\title{
Examining the Hydrological Variations in an Aquaplanet World Using Wave Activity Transformation $\mathscr{O}$
}

\author{
JiAn LU, KoICHI SAKAgUCHI, QING YANG, AND L. RUBy LEUNG \\ Atmospheric Sciences and Global Change Division, Pacific Northwest National Laboratory, Richland, Washington \\ GANG CHEN \\ Department of Atmospheric and Oceanic Sciences, University of California, Los Angeles, Los Angeles, California
}

CHUN ZHAO

Atmospheric Sciences and Global Change Division, Pacific Northwest National Laboratory, Richland, Washington

\section{ERIK SWENSON}

Center for Ocean-Land-Atmosphere Studies, George Mason University, Fairfax, Virginia

\section{ZHANGSHUAN J. HOU}

Atmospheric Sciences and Global Change Division, Pacific Northwest National Laboratory, Richland, Washington

(Manuscript received 29 July 2016, in final form 22 December 2016)

\begin{abstract}
Building on the recent advent of the concept of finite-amplitude wave activity, a contour-following diagnostics for column water vapor (CWV) is developed and applied to a pair of aquaplanet model simulations to understand and quantify the higher moments in the global hydrological cycle. The Lagrangian nature of the diagnostics leads to a more tractable formalism for the transient, zonally asymmetric component of the hydrological cycle, with a strong linear relation emerging between the wave activity and the wave component of precipitation minus evaporation $(\widehat{P-E})$. The dry-versus-wet disparity in the transient hydrological cycle is measured by $\overline{P-E}$, and it is found to increase at a super-Clausius-Clapeyron rate at the poleward side of the mean storm track in response to a uniform sea surface temperature (SST) warming and the meridional structure of the increase can be largely attributed to the change of the meridional stirring scale of the midlatitude Rossby waves. Further scaling for $\overline{P-E}$ indicates that the rate of the wavy hydrological cycle, measured by the ratio of $\overline{P-E}$ to the CWV wave activity, is subdued almost everywhere in the extratropics, implying an overall weakening of the transient circulation. Extending the CWV wave activity analysis to the transient moist regions helps reveal some unique characteristics of atmospheric rivers in terms of transport function, minimum precipitation efficiency, and maximum hydrological cycle rate, as well as an overall weakening of the hydrological cycle rate in the atmospheric river regions under SST warming.
\end{abstract}

\section{Introduction}

Hydrological extremes, such as extended drought and heavy precipitation, can cause disproportional damage to local society and the ecosystem compared to more

Supplemental information related to this paper is available at the Journals Online website: http://dx.doi.org/10.1175/ JCLI-D-16-0561.s1.

Corresponding author e-mail: Jian Lu, jian.lu@pnnl.gov clement hydrometeorological events. Moreover, many climate models projected greater increases in extreme precipitation than that of the mean in many regions of the globe (e.g., Hennessy et al. 1997; Kharin and Zwiers 2000; Wehner 2004; Emori et al. 2005; Pall et al. 2007). Precipitation is a positive definite quantity with a long, thick tail in its probability distribution function (PDF). This nonnormality of PDF leads to confounding behaviors of the precipitation extreme in response to different forcing perturbations. Hence, while considerable inferences can be made with regard to the temperature 
extremes from the knowledge of the change of the mean temperature (e.g., Gao et al. 2015a; Schneider et al. 2015), this is not true for precipitation. A decrease in the mean precipitation does not necessarily imply a decrease at the tail of the precipitation distribution (e.g., Fig. 12.27 in Collins et al. 2013; Pendergrass and Gerber 2016).

To date, most studies have focused on the statistical and descriptive aspects of the extremes (e.g., Kharin et al. 2013). Often only thermodynamic rationales are invoked to explain the increase of the hydrological extremes. Many studies have attempted to link the increase of hydrological extremes to temperature warming through the Clausius-Clapeyron (CC) relation. For instance, it has been argued that under global warming and under the constraint of constant relative humidity (e.g., Ingram 2002; Mitchell et al. 1987; Semenov and Bengtsson 2002) the CC relation implies that the atmospheric moisture would increase exponentially with temperature (approximately $7 \% \mathrm{~K}^{-1}$ ). If extreme precipitation events are to precipitate out all the moisture in the air column, one might expect the uppermost quantiles of the precipitation events to be constrained by the increase in the column water vapor. This reasoning is oversimplified as the efficiency of rainfall mechanisms is relatively low, not all storm-ingested water vapor is converted to precipitation, and it is impossible to dry out the air column completely (e.g., Trenberth et al. 2003). So both dynamics and thermodynamics play indispensable roles in the changes of precipitation extremes in a warmer climate. Thus, the key to understanding the hydrological response to climate change, particularly its spatial dependence, requires understanding of the underlying circulation and dynamics as well. To date, the issue of the precipitation extremes has mostly been approached from the angle of conditional sampling (e.g., Kharin and Zwiers 2000; Lenderink and van Meijgaard 2008), instead of fundamental thermodynamic and dynamic constraints. The lack of dynamic principle-based analysis has limited the inquiries into the dynamical underpinning behind the response of the hydrological extremes to climate change.

Meanwhile, some robust patterns of increase in precipitation extremes have emerged from analysis of the Coupled Model Intercomparison Project (CMIP) simulations. Emori and Brown (2005) and Toreti et al. (2013) found that precipitation extremes in terms of return level increase with much greater magnitude in the mid-to-high latitudes than in the subtropics. Lu et al. (2014a) suggested a dynamically induced poleward shift of the precipitation PDF under SST warming as a mechanism behind the meridional structure and the apparent super-CC rate of increase in the precipitation extremes. In addition, a dynamical modulation on the spatial distribution of atmospheric rivers (ARs) large-scale features characterized by narrow filaments of lower tropospheric water vapor and concentrated water vapor transport in the subtropical-to-midlatitude oceans-has been recently established (Gao et al. 2015b, 2016). However, these attributions of the hydrological extremes to dynamic factors are mostly empirical; more rigorous diagnostic frameworks for quantitative understanding of the hydrological extremes are in demand.

In this study, we strive to develop a diagnostic framework and close budget for an (area) integral property of the transient, zonally anomalous hydrological variations based on the concept of the finiteamplitude wave activity adopted from the field of geophysical fluid dynamics. This allows us to probe the thermodynamic and dynamic signatures on the spatial structure of the water vapor wave activity and the associated hydrological variations and how they might respond to an idealized, pseudowarming perturbation in the SST. The benefits of the formalism for the wave activity budget are manifold. The greatest benefit may be the simplicity in the formalism of the problem, which allows us to investigate the budget for the higher moments of water vapor in a simple mathematical form. The resultant formalism for column water vapor (CWV) wave activity can be readily extended to characterize distinct phenomena such as atmospheric rivers. Indeed, some insights unattainable by conventional analysis can be gained. For instance, we find that not only the time mean, but also the transient, wave component of the hydrological cycle, measured by the rate of removal of moisture through precipitation minus evaporation, should be weakened under global warming and this may be attributed to the dynamical aspect (weakening of the transient motion) of the climate warming.

The paper is organized as follows. We first introduce the model and simulations used for this study in section 2. This is followed by the formulation of the finiteamplitude wave activity budget for CWV in section 3 . The results of the budget and its response to a uniform SST warming are reported in section 4 . Section 5 extends the budget analysis to the transient moist areas to shed light on the key characteristics of the subtropical atmospheric rivers. Finally, the paper concludes with a summary and discussion.

\section{Model and aquaplanet simulations}

The model used for this study is an atmospheric general circulation model known as the Model for Prediction Across Scales (MPAS). Its atmospheric dynamical core is configured on spherical centroidal Voronoi tessellations 


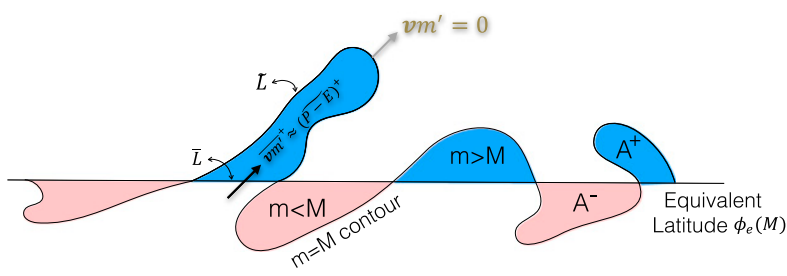

FIG. 1. Schematic diagram for the definition of CWV wave activity. The wavy curve indicates a contour of CWV $(M)$, above which the $m$ values are smaller than below. Inside the red lobes $m<M$ and inside the blue lobes $m>M$. The superscript plus sign indicates the wave activity and budget terms within the moist (blue) lobes. The equation in gray indicates that the flux of $m^{\prime}=$ $m-M$ across the $M$ contour identically vanishes.

(Du et al. 1999; Ringler et al. 2008), making it amenable to local mesh refinement. When configured with variableresolution meshes, both the conservation properties (including energy, mass, tracer, and potential vorticity) and rate of convergence are unchanged (Ringler et al. 2011). However, in the current study, only simulations with quasi-uniform resolutions are analyzed. A more detailed description of the MPAS model and its simulation of the key statistics of the climate in different numerical experiments can be found in Rauscher et al. (2013), O'Brien et al. (2013), Leung et al. (2013), and Sakaguchi et al. (2015). The hydrostatic version of the MPAS dynamical core is coupled with the physics parameterizations of the Community Atmosphere Model version 4 (CAM4), and the major improvement of this physics package compared to previous versions are documented in Neale et al. (2010).

The aquaplanet experiments with MPAS are configured with a global sea surface boundary with no ice and forced by the prescribed SST following the protocol of Neale and Hoskins (2000):

$$
\operatorname{SST}(\phi)= \begin{cases}27^{\circ} \mathrm{C}\left[1-\sin ^{2}\left(\frac{3 \phi}{2}\right)\right], & \text { if }-\frac{\pi}{3}<\phi<\frac{\pi}{3} . \\ 0^{\circ} \mathrm{C}, & \text { Otherwise }\end{cases}
$$

For this control SST condition, experiments at three different resolutions are conducted with mesh sizes approximately 60,120 , and $240 \mathrm{~km}$, respectively. For each simulation, the integration is run for 3 years, with the first 12 months discarded for spinup. In each doubling of the horizontal resolution, all the adjustable parameters in the physics suite are fixed at the standard values except that the $\nabla^{4}$ horizontal diffusion coefficients are reduced to minimize the impact of the numerical diffusion while ensuring numerical stability.

Compared to the MPAS model used in Lu et al. (2015, hereafter L15), the only difference is a bug fix in the interface between the MPAS dynamical core and the CAM4 physics, eliminating a small moisture imbalance between the global precipitation and evaporation. Moreover, this bug fix ensures a close balance between $P-E$ and the vertically integrated moisture convergence. To explore the impact of global warming on the hydrological cycle and extremes, we perform an extra pseudowarming experiment with the 240-km resolution MPAS by adding a 3-K uniform perturbation to the SST boundary condition. The response of the time mean, zonal mean $P-E$ to $3-\mathrm{K}$ warming in MPAS is typical of the aquaplanet response to uniform SST warming [e.g., see Fig. 1 in Chen et al. (2013)], characterized by a sharp increase at the equatorial ITCZ and an expansion of the dry zone (represented by the negative $P-E$ in the subtropics) both poleward and equatorward, with the latter being referred to as the deep-tropics squeeze by Lau and Kim (2015).

\section{Formulation of the wave activity budget for CWV}

The purpose of formulating a wave activity budget for CWV is twofold. First, through the wave activity transformation, the time and zonal mean component (i.e., the first moment) is automatically eliminated to concentrate on the integrated properties of the higher moments of the hydrological cycle. As it turns out, the transformation of $P-E$ can then be linked linearly to the $\mathrm{CWV}$ wave activity through a regression parameter and hence becomes scalable. In fact, an empirical scaling relation for the transformed $P-E$ and its response to SST warming is the main outcome of this study. Second, the CWV wave activity budget can be naturally extended to the water vapor over moist areas (i.e., areas with positive CWV anomalies due to poleward intrusion; blue areas in Fig. 1). The large wave activity events can be associated with the phenomena called atmospheric rivers, which in turn are related to the Rossby wave breaking dynamically (e.g., Lu et al. 2014b; Payne and Magnusdottir 2014). The wave activity budget for the moist area, that is, the moist wave activity budget, can shed fresh light on the unique properties of the atmospheric rivers and how they respond to warming.

We start with the familiar moisture equation

$$
\frac{\partial q}{\partial t}+\mathbf{V} \cdot \nabla q+\omega \frac{\partial q}{\partial p}=e-c,
$$

where $q$ is specific humidity; $e$ and $c$ are evaporation and condensation per unit mass, respectively, which together constitute the precipitation rate in the atmosphere; 
and $\mathbf{V}$ and $\omega$ are the horizontal wind vector and vertical pressure velocity, respectively. A vertical integration of (1) from the top of the atmosphere $(p=0)$ to the surface $\left(p=p_{s}\right)$ gives the budget equation for the CWV $m$ :

$$
\frac{\partial m}{\partial t}+\nabla \cdot \mathbf{F}=E-P
$$

where

$$
\begin{aligned}
& m=\frac{1}{g} \int_{0}^{p_{s}} q d p, \\
& \mathbf{F}=\frac{1}{g} \int_{0}^{p_{s}} q \mathbf{V} d p, \\
& P=\frac{1}{g} \int_{0}^{p_{s}} c d p, \quad \text { and } \\
& E=\frac{1}{g} \int_{0}^{p_{s}} e d p .
\end{aligned}
$$

Note that $P$ and $E$ represent the precipitation and evaporative moisture flux at the surface, respectively, provided that cloud water content is approximately constant for the time scale of interest here. Equation (2) is the same as (6) in Trenberth and Guillemot (1995) and the derivation can be found therein.

We further define a vectorial quantity for velocity

$$
\mathbf{v}=\frac{\mathbf{F}}{m}
$$

which represents water vapor transport normalized by the CWV. Since water vapor is concentrated in the lower troposphere, this velocity reflects the circulation near the boundary. It can be further decomposed into rotational and divergent components:

$$
\mathbf{v}=\mathbf{v}_{\psi}+\mathbf{v}_{\chi}
$$

with $\nabla \cdot \mathbf{v}_{\psi}=0$. Noting that

$$
\nabla \cdot \mathbf{F}=\nabla \cdot\left(\mathbf{v}_{\psi} m+\mathbf{v}_{\chi} m\right)
$$

we can rewrite (2) as

$$
\frac{\partial m}{\partial t}+\mathbf{v}_{\psi} \cdot \nabla m=E-P-\nabla \cdot\left(\mathbf{v}_{\chi} m\right)-R .
$$

The additional term $R$ represents the numerical dissipation of $\mathrm{CWV}$ in the model, reflecting the diffusive effect of the third-order transport scheme on scalars used in MPAS (Skamarock and Gassmann 2011). Except for the extra $E-P$ term, (3) has a similar form as the vorticity equation in the pressure coordinate [see (3.1) in L15].
To construct the CWV wave activity budget, we adopt the area-coordinate transformation similar to Nakamura and Zhu (2010)

$$
\Delta \mathcal{A}() \equiv-\frac{1}{2 \pi a \cos \phi_{e}}\left[\iint_{m<M}() d S-\int_{\phi>\phi_{e}}() d S\right],
$$

where $a$ donates the radius of Earth and $M$ is the value of a wavy, instantaneous CWV contour with a corresponding equivalent latitude $\phi_{e}$. The equivalent latitude $\phi_{e}$ for a given $M$ value (upper case is used to emphasize its Lagrangian property) is determined uniquely by the requirement that the area enclosed by the $M$ contour toward the polar cap equals the area poleward of latitude $\phi_{e}$, that is,

$\int_{m<M, \phi<\pi / 2} d S=\iint_{\phi_{e}<\phi<\pi / 2} d S=2 \pi a^{2}\left(1-\sin \phi_{e}\right)$.

For a given equivalent latitude $\phi_{e}$, the net effect of the two integral operators in (4) is the difference of the integration over the areas enclosed by the $M$ contour poleward of $\phi_{e}$ (blue areas) minus that equatorward of $\phi_{e}$ (red areas) as illustrated in Fig. 1 (see also Nakamura and Zhu 2010 and L15). Note here that Northern Hemisphere $(\mathrm{NH})$ conventions are chosen and since CWV decreases with latitude in the $\mathrm{NH}, m<M$ is used to define the polar cap area. The sign should be adjusted accordingly in the Southern Hemisphere.

Applying operator $\Delta \mathcal{A}$ to $m$, we can define the finiteamplitude wave activity for $\mathrm{CWV}$ in the $\mathrm{NH}$ as

$$
\begin{aligned}
A\left(\phi_{e}, t\right) & \equiv \Delta \mathcal{A}(m) \\
& \equiv \frac{1}{2 \pi a \cos \phi_{e}}\left(\iint_{m>M, \phi>\phi_{e}} m d S-\iint_{m<M, \phi<\phi_{e}} m d S\right) .
\end{aligned}
$$

For compactness, hereafter we use () to replace the area-coordinate transformation $\Delta \mathcal{A}()$. Then the CWV wave activity equation can be written as

$$
\frac{\partial A}{\partial t}-\overline{v_{\psi}^{\prime} m^{\prime}}=\left[\overline{(E-P)}-\overline{\nabla \cdot\left(\mathbf{v}_{\chi} m\right)}\right]-\tilde{R},
$$

where the overbar indicates zonal mean and prime denotes the deviation from the zonal mean. All the terms except the second term on the left-hand side are a function of equivalent latitude $\phi_{e}$; however, these Lagrangian terms are balanced by the Eulerian term evaluated at latitude $\phi=\phi_{e}$. Therefore, (7) constitutes a hybrid Eulerian-Lagrangian budget system. Again, (7) is similar to its vorticity counterpart as expressed by (3.6) in L15 except for the additional $\overline{(E-P)}$ term, which is the major sink of the equation. Readers are 
referred to the appendix of L15 for the derivation of the terms in (7). The second term on the left-hand side is simply the zonal mean advective CWV flux by $\mathbf{v}_{\psi}$. The second term on the right-hand side represents the convergence of moisture by the divergent velocity component $\mathbf{v}_{\chi}$, serving as a major source of CWV wave activity. The third term is the numerical dissipation of CWV wave activity and it can be calculated as a residual. As it turns out, $-\tilde{R}$ in steady state is always negative for all equivalent latitudes and it decreases in magnitude with increasing model resolution as the model can resolve more moisture structures at finer scales and the balance between the total moisture convergence and $\overline{(E-P)}$ holds more accurately.

Alternatively, without decomposing the CWV flux velocity into divergent and rotational components, (7) can also be written as

$$
\frac{\partial A}{\partial t}=[\overline{(E-P)}-\overline{\nabla \cdot(\mathbf{v} m)}]-\tilde{R}
$$

with $\overline{v_{\psi}^{\prime} m^{\prime}}$ and $\overline{\nabla \cdot\left(\mathbf{v}_{\chi} m\right)}$ collapsing into $\overline{\nabla \cdot(\mathbf{v} m)}$. Equation (8) will be utilized for the wave activity budget for the moist areas in section 5 .

The advantage of the $\Delta \mathcal{A}$ transformation may be further appreciated by comparing (7) to its Eulerian counterpart. By defining an Eulerian CWV wave activity (positive in Northern Hemisphere)

$$
A^{E} \equiv-\left(\frac{1}{a} \frac{\partial \bar{m}}{\partial \phi}\right)^{-1} \frac{\overline{m^{\prime 2}}}{2}
$$

the corresponding budget equation can be derived by multiplying the CWV zonal perturbation equation by $m^{\prime}$ and dividing it by $a^{-1} \partial \bar{m} / \partial \phi$ :

$$
\begin{aligned}
\frac{\partial A^{E}}{\partial t} & -\overline{v^{\prime} m^{\prime}}-\left(\frac{1}{a} \frac{\partial \bar{m}}{\partial \phi}\right)^{-1}\left[\frac{1}{a} \frac{\partial}{\partial \phi}\left(\overline{\frac{v m^{\prime 2}}{2}}\right)+\frac{\bar{m}}{a} \frac{\partial}{\partial \phi}\left(\overline{v^{\prime} m^{\prime}}\right)\right] \\
= & -\left(\frac{1}{a} \frac{\partial \bar{m}}{\partial \phi}\right)^{-1}\left[\overline{m^{\prime}(E-P)^{\prime}}\right] .
\end{aligned}
$$

One can see that (10) is much more cumbersome, involving cubic terms, and the related interpretation can be convoluted, whereas the wave activity expressed in (7) or (8) preserves the intuition of the original moisture budget and is more amenable to interpretation. From (10), one can also see that $\overline{(E-P)}$ plays the same role as $-\left(a^{-1} \partial \bar{m} / \partial \phi\right)^{-1}\left[\overline{m^{\prime}(E-P)^{\prime}}\right]$ in (10) in the small perturbation limit that neglects the nonlinear cubic term.

The actual budget of (7) is computed using the model output of velocity, specific humidity, precipitation, and evaporation averaged every $6 \mathrm{~h}$ in the last two years of the simulation.

\section{Result of CWV wave activity budget}

\section{a. Control run}

Two snapshots of the distribution of CWV are illustrated in Fig. 2 for a large and a small wave activity at equivalent latitude $20^{\circ} \mathrm{N}$, respectively. It is evident that large wave activity is associated with a large excursion of the $M$ contour, large poleward transport of moisture indicated by the flux vectors, and large amount of precipitation near the tip of the curvy $M$ contour, features often associated with atmospheric rivers that transport moisture over a long distance. But compared to real rivers, the atmospheric rivers are much more leaky, with substantial amount of moisture lost to precipitation along the way. Estimating the efficiency of the moisture transport by the meridional moist intrusions is one of the major outcomes of this budget analysis. The largest AR event in Fig. 2a (the second wave from the west) appears to tap moisture from the deep tropics, consistent with the notion that equatorial waves can sometimes organize the tropical moisture for the atmospheric rivers to draw on (Bao et al. 2006). It is worth noting that despite the large variation in the wave activity between the quiescent and active phases, for a given $M$ value the corresponding equivalent latitude changes little (usually no more than $2^{\circ}$ latitude; see the animation in the supplemental material for further confirmation).

From the animation in the supplemental material, one also notices that for a given subtropical equivalent latitude (and $M$ ) strong precipitation occurs at the poleward tip of the moist plumes, while the precipitation intensity is weak near equivalent latitude. The latitudinal distributions of the positive CWV anomalies $(m>M)$ and precipitation are elucidated in Fig. 3, which shows that, away from the equivalent latitude, positive moist anomalies occur less frequently (blue bars), whereas the precipitation per unit area (shading) first increases with latitude and can peak as far as over a thousand kilometers poleward of the equivalent latitude.

The CWV wave activity $A$ may be thought of as arising from kinematic stirring (because of baroclinic waves and wave breaking) of a monotonically distributed background CWV imposed by the thermodynamic factors of the aquaplanet, but subject to the modulation by condensation and evaporation (e.g., O'Gorman and Schneider 2006). The zonal mean and 

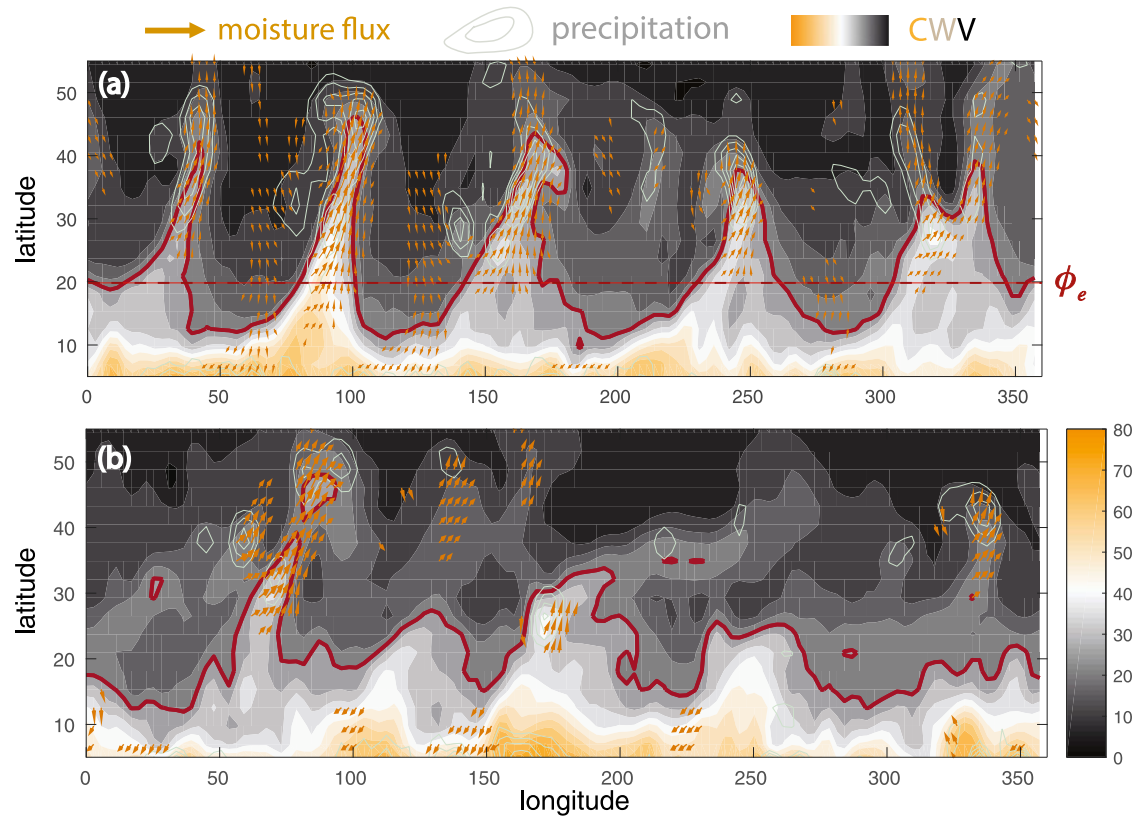

FIG. 2. A snapshot of CWV (shading; kg), precipitation (contours; contour interval is $18 \mathrm{~mm} \mathrm{day}^{-1}$ ), and integrated moisture flux with the meridional component $>140 \mathrm{~kg} \mathrm{~m} \mathrm{~s}^{-1}$ (arrows) for (a) a large wave activity and (b) a low wave activity case. The thick red line demarcates the $M$ contour corresponding to $\phi_{e}=20^{\circ} \mathrm{N}$ (indicated by the dashed red line) in (a).

the Lagrangian mean negative meridional gradient of $\mathrm{CWV}$ as a function of latitude (or equivalent latitude for the latter) are shown in Fig. $4 \mathrm{~b}$ as the thin and thick lines, respectively. Both gradients are negative throughout the $\mathrm{NH}$, indicative of monotonicity in the mean CWV distribution. Interestingly, the Eulerian gradient has a dip at the latitude $\left(\sim 25^{\circ}\right)$ where wave activity is maximized (Fig. 4a). This feature is absent in the Lagrangian gradient, which is in better agreement with what the $\mathrm{CC}$ relation would predict from the SST boundary condition. By the construction of the CWV wave activity, the zonal mean gradient and the Lagrangian gradient is linked through (see Nakamura and Zhu 2010)

$$
\frac{\partial \bar{m}}{\partial y}=\frac{\partial M}{\partial y}+\frac{\partial^{2}\left(A \cos \phi_{e}\right)}{\partial y^{2}}, \quad y \equiv a \sin \phi_{e}
$$

Therefore, the deviation of $\partial \bar{m} / \partial y$ from a smooth background profile $\partial M / \partial y$, which is determined by the prescribed thermodynamic conditions, can be attributed to the waviness embedded in the moisture field. The actual meridional structure of $A$ in equilibrium is the result of a delicate balance among evaporation $\tilde{E}$, precipitation $\tilde{P}$, divergent flux across the $M$ contour and the corresponding equivalent latitude $\overline{\nabla \cdot\left(\mathbf{v}_{\chi} m\right)}$, rotational advection across the equivalent latitudes $\overline{v_{\psi}^{\prime} m^{\prime}}$, and numerical dissipation $\tilde{R}$. Both the CWV wave activity (Fig. 4a) and its standard deviation (Fig. 5a) peak at approximately $25^{\circ}$ latitude equatorward of the mean jet, which is at approximately $36^{\circ}$ latitude for this case of MPAS simulation at $240-\mathrm{km}$ resolution. This is consistent with the fact that atmospheric rivers are often observed on the equatorward flank of the jet and

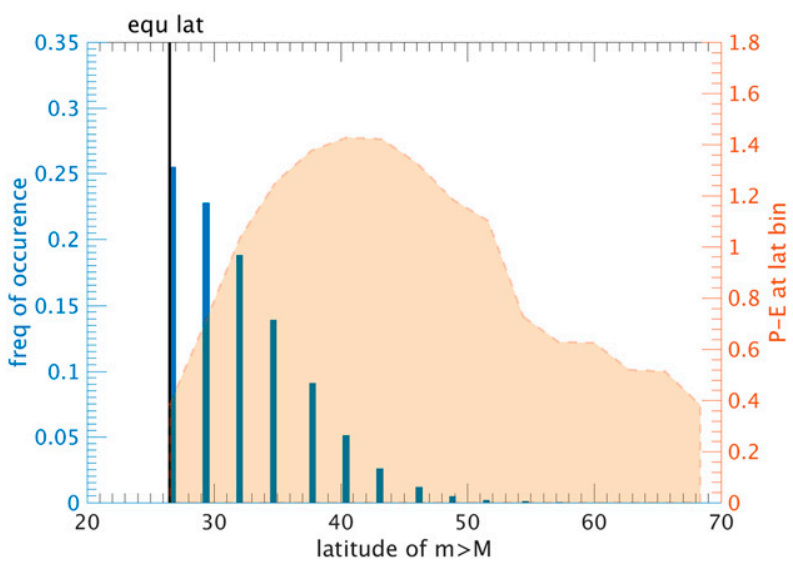

FIG. 3. Left $y$ axis shows the frequency of occurrence of $m>M$ in the moist areas at the poleward of the equivalent latitude $26.5^{\circ} \mathrm{N}$ (blue bars). Right $y$ axis shows the corresponding averaged $P-E$ (cm day $^{-1}$ ) during the occurrence of $m>M$ at each latitude poleward of the equivalent latitude $26.5^{\circ} \mathrm{N}$ (sand-colored shading). (Note that $M=16.45 \mathrm{~kg}$ is the Lagrangian CWV value corresponding to equivalent latitude $26.5^{\circ} \mathrm{N}$.) 

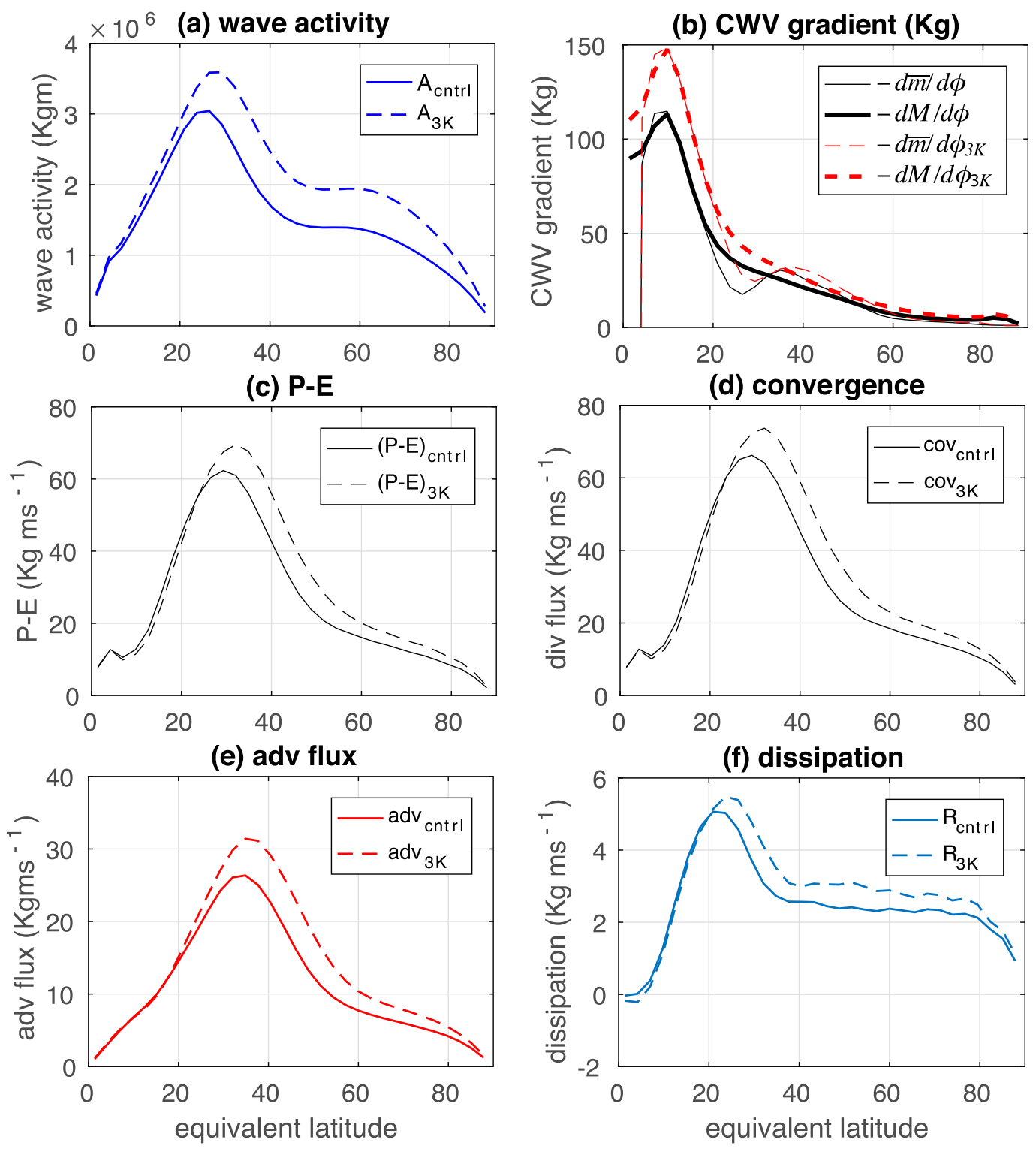

FIG. 4. Result of CWV wave activity budget for the control (solid line) and 3-K pseudowarming (dashed line) MPAS simulations: (a) $A$; (b) $-d \bar{m} / d \phi$ and $-d M / d \phi$; (c) $\overline{(P-E)}$; (d) total convergence $-\overline{\nabla \cdot(\mathbf{v} m)}=$ $\overline{v_{\psi}^{\prime} m^{\prime}}-\left[\overline{\nabla \cdot\left(\mathbf{v}_{\chi} m\right)}\right]$; (e) advective flux $\overline{v_{\psi}^{\prime} m^{\prime}}$; and (f) dissipation as residual.

characteristic of large elongated moisture transport anomalies connecting from the tropics to the midlatitudes (Zhu and Newell 1998), as well as the interpretation that ARs are largely associated with the anticyclonic wave breakings there (Payne and Magnusdottir 2014). Interestingly, the maxima of the mean and the variance of wave activity collocate with the minimum of the skewness (Fig. 5b). The skewness is less than 0.5 equatorward of $50^{\circ}$ latitude, and increases with latitude in general. The latter may not reflect the true statistics of the wave activity in the polar region, but the decreasing sample size as one approaches the pole (keep in mind that the grid cells are uniform in size in MPAS).

For the budget of $A$, both the advection across the latitude $\phi_{e}\left(\overline{v_{\psi}^{\prime} m^{\prime}}\right.$; Fig. 4e) and convergent flux across the $M$ contours $\left[-\overline{\nabla \cdot\left(\mathbf{v}_{\chi} m\right)}\right.$; not shown] contribute positively and roughly equally to the budget, with their sum $[-\overline{\nabla \cdot(\mathbf{v} m)}]$ being approximately twice as large (Fig. 4d). Similar to the conventional moisture budget, a large majority of $-\overline{\nabla \cdot(\mathbf{v} m)}$ turns into $\overline{(P-E)}$ (cf. Figs. 4c and 3d), with the small residual as numerical dissipation (Fig. 4f). The dissipation term is positive definite for all latitudes, indicative of a 

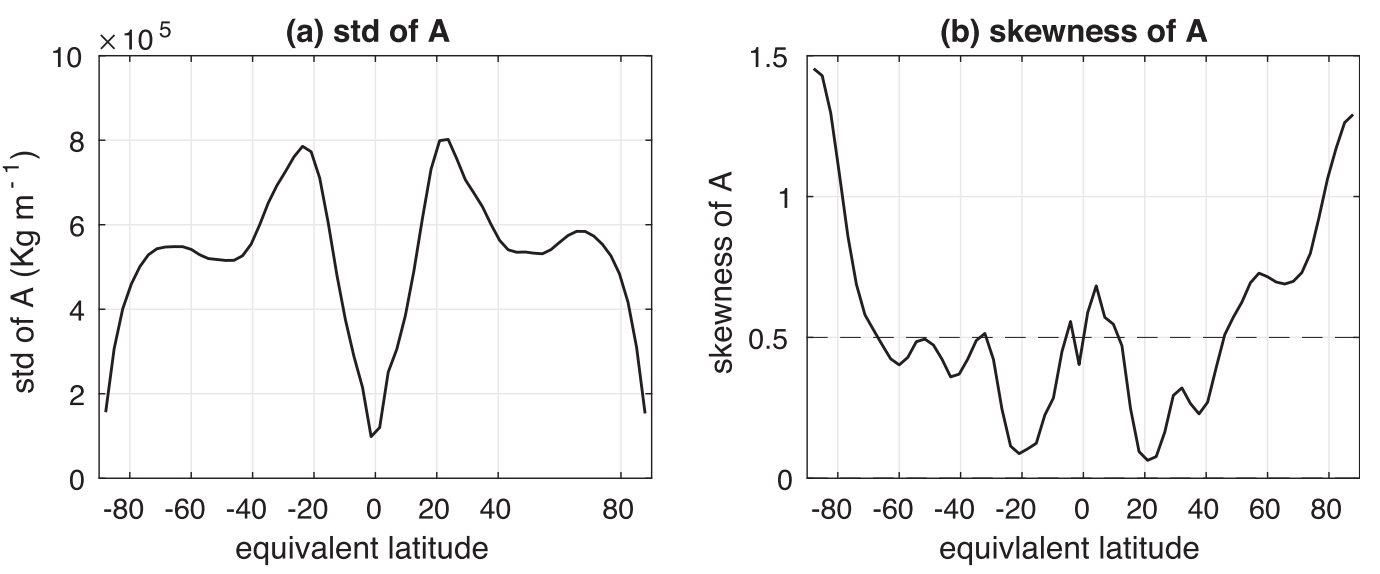

FIG. 5. (a) Standard deviation and (b) skewness of $A$ in the control simulation.

numerical sink of moisture variance. The leadingorder balance for the CWV wave activity budget presents a stark contrast with the wave activity budget for upper tropospheric vorticity. As shown in L15, the wave source and dissipation are both larger in magnitude than the advection term for the budget of vorticity wave activity, whereas here the dissipation term is an order smaller than the advection and sink/ source terms. As such, the CWV tracer should be better characterized as an advection-condensation problem rather than an advection-diffusion problem for more conservative tracers. Subsequently, the asymptotic behavior of the effective diffusivity demonstrated in L15 for the tracer subject to advection and diffusion within the Batchelor regime (e.g., Haynes 2010) cannot be directly applied to the CWV wave activity here.

The meaning of $\overline{(P-E)}$ demands further explanation. As shown in the schematic Fig. 1, it is the difference between the area integral of $P-E$ over high moisture anomalies (blue areas) and that over low moisture anomalies (red areas), thus indicating dryversus-wet disparity of the hydrological cycle. Moreover, the area coordinate transformation for wave activity emphasizes the contribution of the large perturbations, weighting the quantity subject to operator $\Delta \mathcal{A}($ ) with a length scale $\eta$, which represents the displacement of an air parcel from its equilibrium position. As such, $\overline{(P-E)}$ captures the higher moments of the zonally asymmetric hydrological variability. Under the limit of small and/or linear perturbation, it can be shown that $\left.\lim _{\eta \rightarrow 0} \overline{(P-E)}=-\left(a^{-1} \partial \bar{m} / \partial \phi\right)^{-1} \overline{\left[m^{\prime}(P-E)^{\prime}\right.}\right]$ (see Nakamura and Solomon 2010), which is the sink of $A^{E}$ in (10). The fact that $\overline{(P-E)}$ is positive implies a positive correlation between $P-E$ and $C W V$, consistent with the CWV snapshot in Fig. 2 in which precipitation is associated with the poleward intrusion of anomalous moist air.

\section{b. 3-K warming and scaling for the change of $\overline{(P-E)}$}

Under 3-K pseudowarming, the CWV wave activity intensifies everywhere across the globe (Fig. 4a). However, the increase is not uniform, but has a strong meridional structure. The feature of a much greater increase poleward relative to equatorward of the mean jet is shared by all the budget terms in (7). More interestingly, the fractional change in $(P-E)$ features a considerable meridional variation, with a decrease (an increase) equatorward (poleward) of $25^{\circ}$ latitude and a super-CC peak (centered around $48^{\circ}$ latitude) poleward of the mean jet (Figs. 4c and 8a). Despite the simplicity of the SST warming, this bears a resemblance to the meridional structures of the change of precipitation extremes found in the comprehensive climate models under future warming scenarios (Toreti et al. 2013; Emori and Brown 2005).

A notable outcome of the area-coordinate transformation on the CWV equation is that the probability distributions of the resultant wave activity and $\overline{(P-E)}$ are close to Gaussian. Figure 6 shows the scatter between the two quantities at $35^{\circ} \mathrm{N}$ and their corresponding histograms. A Shapiro-Wilk test (Royston 1982) indicates that the PDF of $\overline{(P-E)}$ is close to normal with a $p$ value of 0.24 (note that the null hypothesis of the Shapiro-Wilk test is the normality of the PDF). The normality of $\overline{(P-E)}$ can be thought of as the result of the central limit theorem as it is a summation of many samples of $P-E$ over a broad area enclosed between the CWV contour and its corresponding equivalent latitude around the globe. The tendency toward normal distribution of wave activity and $\overline{(P-E)}$ can significantly simplify the investigation of the hydrological 


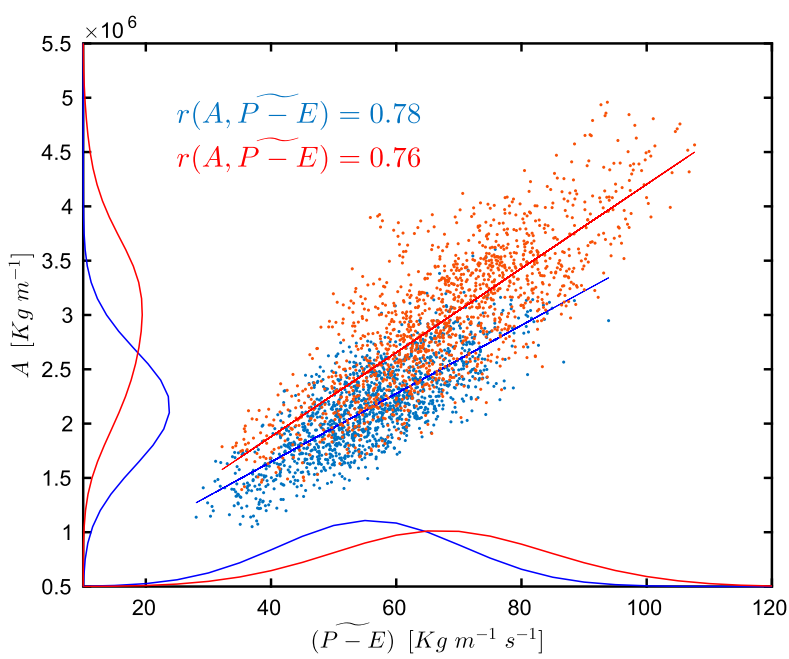

FIG. 6. The scatter of 6-hourly $A$ against $\overline{(P-E)}$ at $35^{\circ} \mathrm{N}$ and the corresponding normalized histograms (curves) from the control (blue) and 3-K (red) MPAS simulations. The correlations between $A$ and $\overline{(P-E)}$ are also labeled.

extremes in the sense that only the first two moments, mean and variance, are needed to capture the full distribution of a Gaussian variable. From Fig. 6, one can see that both the mean and the standard deviation of $A$ and $\overline{(P-E)}$ increase under $3-\mathrm{K}$ SST warming. Furthermore, the close linear relationship between them serves as an important constraint for the scaling exercise that follows.

A simple regression of $A$ against $\overline{(P-E)}$, together with the resultant near-zero intercepts, leads to a scaling relation for the latter:

$$
\overline{(P-E)} \sim \frac{A}{\tau}
$$

For the transient variability of $A, \tau$ can be interpreted as the time scale at which $\overline{(P-E)}$ acts as a wave activity sink to restore the wave activity toward a wavefree reference state $(A=0)$. A scale analysis can relate the time mean $\overline{(P-E)}$ approximated to $\overline{v m^{\prime}}$ (see the reasoning in section 5 and the appendix). With $m^{\prime} \sim-\eta \partial M / \partial y, \overline{(P-E)}$ can be scaled as $-K \partial M / \partial y$, where $K \sim v \eta>0$ is an eddy diffusivity. If we further assume that $v \sim(2 \tau)^{-1} \eta$ (considering that at equilibrium the growth and decay of $\eta$ should be balanced), representing a correction on $v$ to account for the damping of the meridional displacement of the contour by the moisture sink $\overline{(P-E)}$, then $\overline{(P-E)} \sim-K \partial M / \partial y \sim$ $-(2 \tau)^{-1} \eta^{2} \partial M / \partial y$. Together with the dimensional scaling for the wave activity, $A \sim-0.5 \eta^{2} \partial M / \partial y$, we yield relation (11) again, but now for the equilibrium mean quantities. The profiles of $\eta$ from the control and $3-\mathrm{K}$ warming runs are displayed in Fig. 7a. The equatorward anticyclonic wave breakings and the poleward cyclonic wave breakings are manifested in the two peaks of $\eta$ at the flanks of the mean jet, while the mean jet location coincides with the $\eta$ minimum, suggestive of its mixing barrier effect. It is especially worth noting that the time scale $\tau$ obtained from the regression (Fig. 7b) is very close to the ratio of mean $A$ to mean $\overline{(P-E)}$ in the model simulations examined, reinforcing that the time scale $\tau$ is an intrinsic parameter to the transient hydrological cycle. As such, $\tau$ in (11) has dual physical interpretations: besides the meaning of the restoration time scale for the transient wave activity, for the equilibrium mean state, $\tau$ also represents the residence time of the moisture anomalies associated with the mean wave activity $[A]$. Therefore, for both transient variability and the mean state, $1 / \tau$ measures the recycling rate,
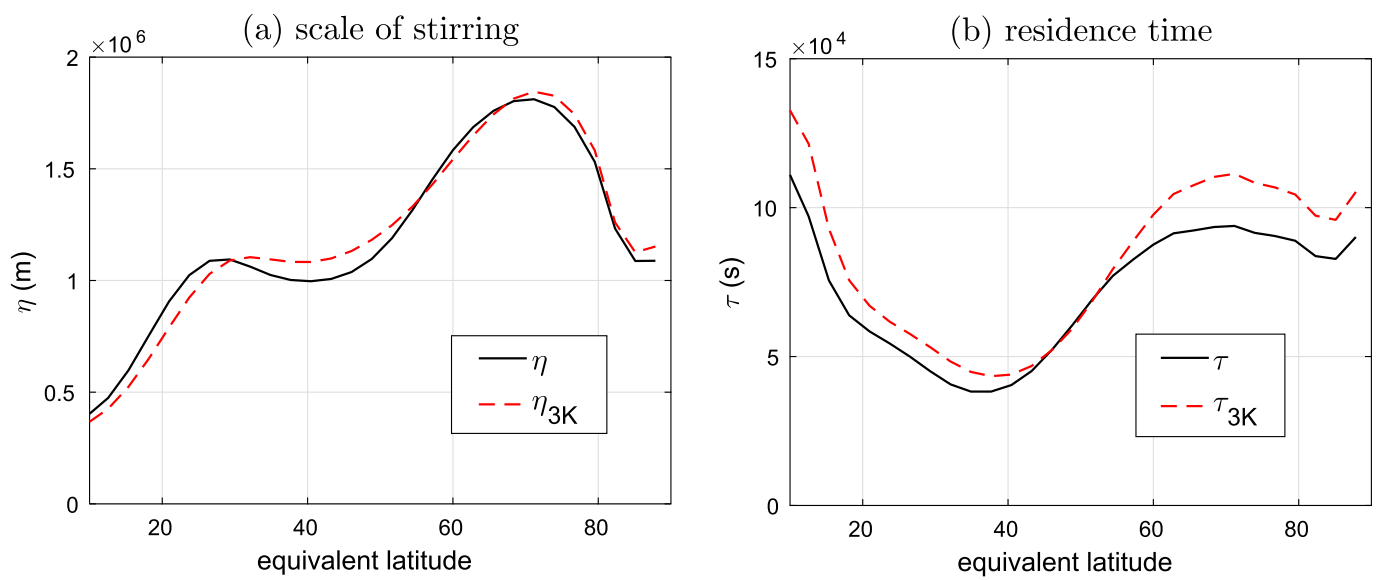

FIG. 7. The meridional distribution of (a) scale of stirring $\eta$ and (b) residence time $\tau$ in the control (black solid line) and 3-K warming (red dashed line) MPAS simulations. 
or intensity, of the wave component of the hydrological cycle. The $\tau$ profile of the control simulation as shown in Fig. $7 \mathrm{~b}$ (black line) exhibits a minimum of about 0.5 day at the peak latitude of the mean jet and storm activity, indicating the vigor of the transient hydrological cycle in the midlatitude storm track.

How would relation (11) vary under a warmer SST condition? Linearizing the time mean of (11) with respect to the control values yields the expression for the fractional change of the wavy hydrological cycle:

$\frac{\delta \overline{(P-E)}}{\overline{(P-E)}} \approx \frac{\delta A}{A}+\frac{\delta \tau^{-1}}{\tau^{-1}} \approx \frac{\delta \partial M / \partial y}{\partial M / \partial y}+\frac{2 \delta \eta}{\eta}+\frac{\delta \tau^{-1}}{\tau^{-1}}$.

In the second approximation, a scaling for the wave activity, $A \sim-0.5 \eta^{2} \partial M / \partial y$, is used and $\eta$ is estimated through $\left[2 A(\partial M / \partial y)^{-1}\right]^{1 / 2}$. Note here all the quantities are time mean or estimated from the time mean values. Thus, the fractional change of $\overline{(P-E)}$ (shown in Fig. 8a) can be attributed to those of hydrological cycle intensity (Fig. 8e) and CWV wave activity (Fig. 8b), with the latter being further factored into the change of the Lagrangian CWV gradient $\partial M / \partial y$ (the thermodynamic factor; Fig. 8d) and the change of the scale of meridional stirring $\eta$ (the dynamic factor; Fig. $8 \mathrm{c}$ ). The immediate outcome of the scaling-probably the most important result of this study-is that the rate of the hydrological cycle weakens almost everywhere except a small latitudinal range poleward of the mean jet (Fig. 8e). This echoes the well-established notion of the weakening of the global mean hydrological cycle intensity (measured by $\langle P\rangle /\langle m\rangle$, where the angle brackets indicate global average). However, the weakening here is regarding the transient wave part of the hydrological cycle, thus more intimately related to the extremes (i.e., droughts and deluges) of the hydrological cycle.

Note that $\eta$ is the main dynamic factor and arguably the most dominant factor in determining the meridional profile of the change of $\overline{(P-E)}$. It is interesting to note that the structure of the $\eta$ changes resembles what one might construct from compositing the poleward shift of the anticyclonic wave breaking, the reduction of the cyclonic wave breaking at the poleward side of the jet, and weak increase of cyclonic wave breaking in high latitudes-a recurrent pattern of wave breakings associated with the poleward shift of the jet found in both simple dry AGCM under idealized heating ( $\mathrm{Lu}$ et al. 2014b; C. Liu and E. Barnes 2016, unpublished manuscript) and comprehensive climate models (Rivière 2011; Barnes and Hartmann 2012) under increasing greenhouse gas forcing. The thermodynamic factor $\partial M / \partial y$ increases at an overall super-CC rate $\left(\sim 10 \% \mathrm{~K}^{-1}\right)$, with a considerable meridional structure (Fig. 8d). The rate of increase of CWV in the extratropics should be better thought of as being set by the warming rate of the air temperature at the last saturation (e.g., Pierrehumbert and Roca 1998; Galewsky et al. 2005; Wright et al. 2010; O'Gorman and Muller 2010), rather than by the warming of the local surface temperature. The superCC rate of increase of $\partial M / \partial y$ reflects the fact that the tropospheric temperature (where last saturation occurs) warms more than the sea surface temperature in both absolute and fractional sense. The evident opposite spatial correlation between the changes in stirring scale $\eta$ and $\partial M / \partial y$ suggests a dynamical modulation on the structure of $\partial M / \partial y$ : an enhanced stirring (as indicated by a larger $\eta$ ) acts to flatten the gradient of CWV and vice versa. Taken together, this scaling result underlines the importance of the dynamical stirring in shaping the meridional distribution of the midlatitude hydrological extremes under global warming.

The reason behind the weakening of the transient hydrological cycle is intriguing, warranting further investigation. The contrast between the profiles of $\delta A / A$ and $\delta(\overline{P-E)} /(\overline{(P-E)}$ reminds us of the reduction of the intensity of the mean hydrological cycle measured by the ratio of the mean precipitation to mean column water vapor, a consequence of overall weakening of the mean atmospheric overturning circulation, which in turn has been rationalized by the constraint that the rate of change of radiative cooling is outpaced by the change of the column moisture (e.g., Allen and Ingram 2002; Held and Soden 2006). This prompts us to compute $\Delta \mathcal{A}$ transformation of the negative pressure velocity at $850 \mathrm{hPa}-\omega_{850}$ (i.e., $\left.-\overline{\omega_{850}}\right)$ to evaluate the transient vertical motion moving along with the eastward traveling moisture waves. As $\omega_{850}<0$ (upward) in the wet lobes and $\omega_{850}>0$ (downward) in the dry lobes, the computed $-\overline{\omega_{850}}$ are positive definite at all equivalent latitudes. The profiles of $-\widehat{\omega_{850}}$ for the control (gray) and $3-\mathrm{K}$ (red) MPAS simulations are shown in Fig. 9, respectively. Comparing the $3-\mathrm{K}$ profile with the control one, there is an evident poleward shift. To elucidate the change in strength, the control profile is shifted poleward by $2.75^{\circ}$ latitude (black dashed), an amount that minimizes the root-mean-square deviation between the two profiles. It is clear that the $3-\mathrm{K}-\widetilde{\omega_{850}}$ is weaker than the shifted control $-\widetilde{\omega_{850}}$ almost everywhere except around $50^{\circ}$ latitude, bearing considerable resemblance to the change of $\tau^{-1}$ in Fig. 8e. As such, the weakening of the wavy hydrological cycle 
(a) $\delta(\widetilde{P-E}) /(\widetilde{P-E})$

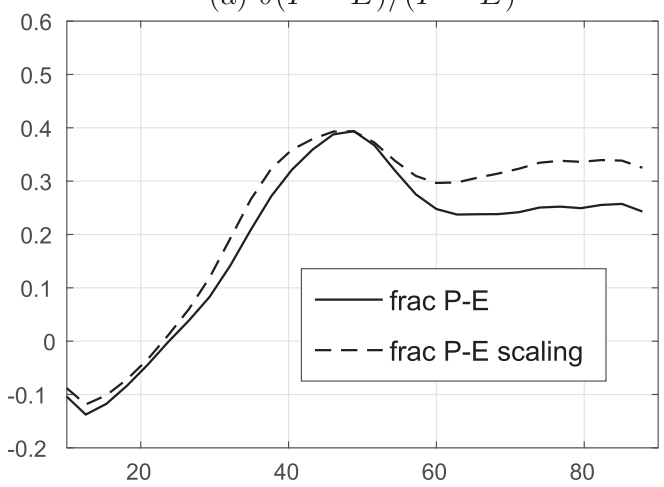

(c) $2 \delta \eta / \eta$

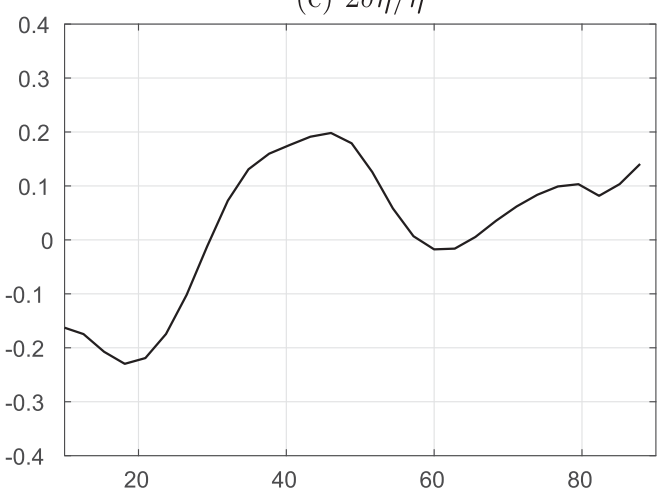

(e) $\delta \tau^{-1} / \tau^{-1}$

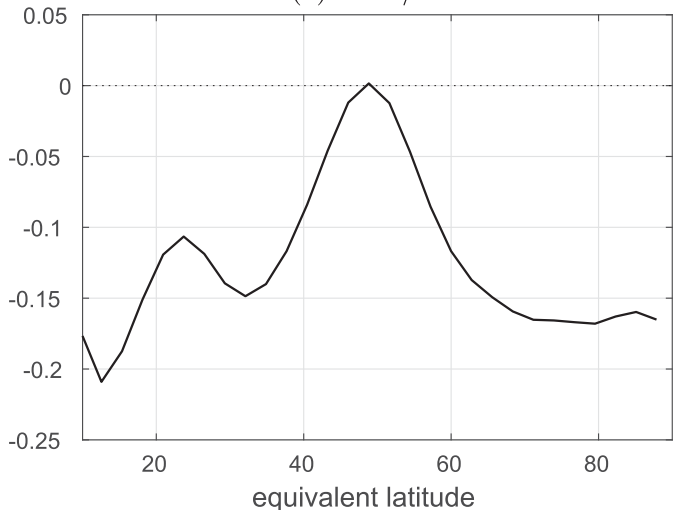

(b) $\delta A / A$

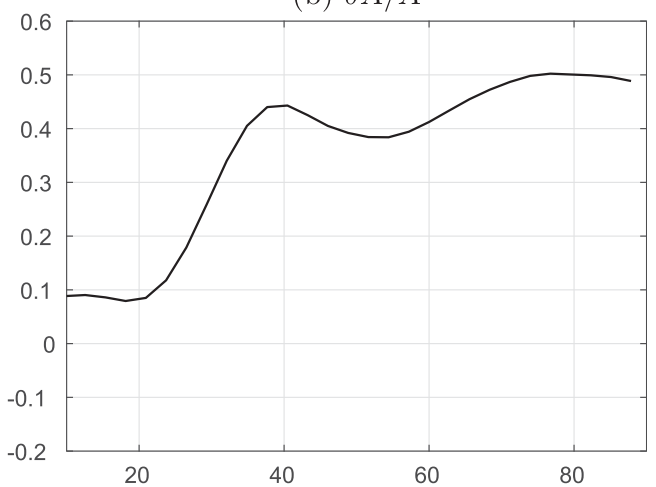

(d) $\delta(d M / d y) /(d M / d y)$

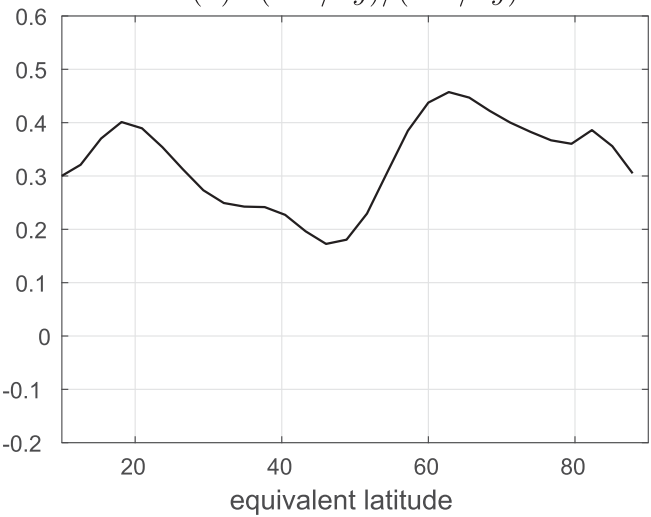

FIG. 8. Result of scaling for the fractional change of $\overline{(P-E)}$ in response to 3-K SST warming: (a) $\delta \overline{(P-E)} / \overline{(P-E)}$ (solid black line) and its scaling based on (12) [dashed black line; i.e., the sum of (c), (d), and (e)]; (b) $\delta A / A$; (c) $2 \delta \eta / \eta$; (d) $\delta(d M / d y) /(d M / d y)$; and (e) $\delta \tau^{-1} / \tau^{-1}$.

intensity is attributable at least partly to the weakening of the transient overturning circulation.

\section{Budget for the moist area with implications for atmospheric rivers}

The wave activity analysis above concerns both the moist and dry areas and cannot discern how much of the increase in $\overline{(P-E)}$ is related to more extreme precipitation and how much to more droughts. For those interested in precipitation and atmospheric rivers, it is more relevant to examine the wave activity budget only over the wet areas where precipitation takes place. To this end, we modify the wave activity budget in the foregoing section to focus only on the moist areas (illustrated as the blue areas in the schematic Fig. 1) whereby to shed light on the moisture budget following moisture features such as atmospheric rivers. 


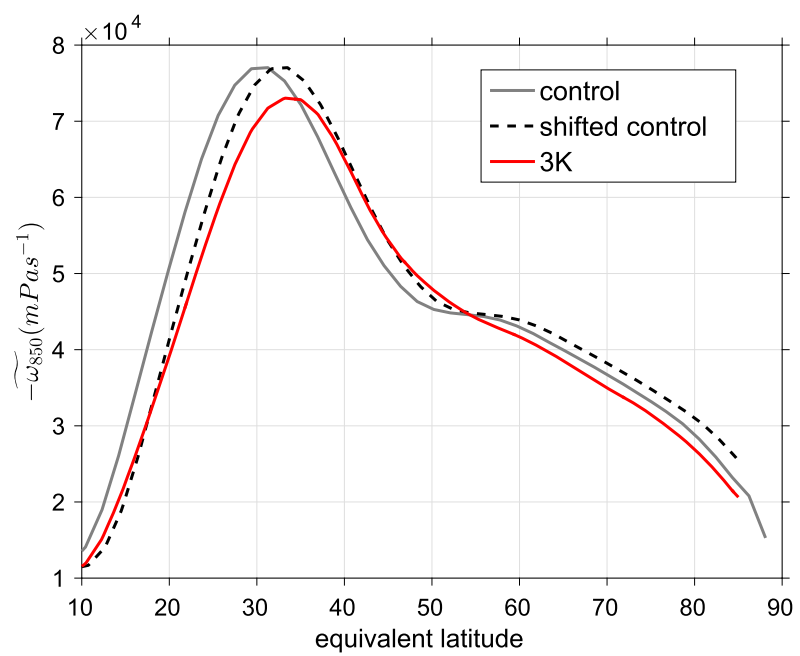

FIG. 9. Shown is $-\widetilde{\omega_{850}}$ for the control (gray line) and 3-K (red line) MPAS simulations measuring the transient overturning circulation moving along with the moisture waves. The black dashed line is the control profile shifted by $2.75^{\circ}$ to minimize the rootmean-square deviation between the control and $3-\mathrm{K}$ profiles.

To focus on poleward moisture intrusion, we define an area integral only over the moist areas as

$$
\Delta \mathcal{A}^{+}() \equiv \frac{1}{2 \pi a \cos \phi_{e}} \iint_{m>M, \phi>\phi_{e}}() d S
$$

and apply it to (3). A simple manipulation by using divergence theorem leads to a budget equation for the moist wave activity $A^{+}$:

$$
\frac{\partial A^{+}}{\partial t}-\overline{v m}^{+}=\overline{(E-P)}^{+}-\frac{1}{2 \pi a \cos \phi_{e}} \int_{\tilde{L}}(\mathbf{v} m) \cdot \mathbf{n} d l,
$$

where $\overline{(~}^{+}$denotes the integration over the longitude segments $\bar{L}$, and where the positive $m-M$ lobe intercepts with the equivalent latitude, normalized by $2 \pi a \cos \phi_{e}, \tilde{L}$ denotes the curvy edge of the positive $m-M$ lobe, and $\mathbf{n} \equiv-\nabla m /|\nabla m|$ is the unit vector normal to $\tilde{L}$. For AR-like filamentary features, $\bar{L} \ll \tilde{L}$ and $\bar{L}$ can be thought of as the base through which water vapor flows into the atmospheric river in subject and the influx water vapor may be considered the "headwater" of the corresponding atmospheric river. In equilibrium, (14) quantifies the budget for the net $P-E$ within the moist areas in terms of contributions from the moisture influx across $\bar{L}$ and outflux across $\tilde{L}$. A simple manipulation of (14) (as elaborated in the appendix) suggests that $\overline{(E-P)}^{+}$is largely balanced by the flux of $m^{\prime}=m-M$ across $\bar{L}$ (i.e., $\overline{v m^{\prime}}{ }^{+}$) in equilibrium, with the part $v M$ only passing through the

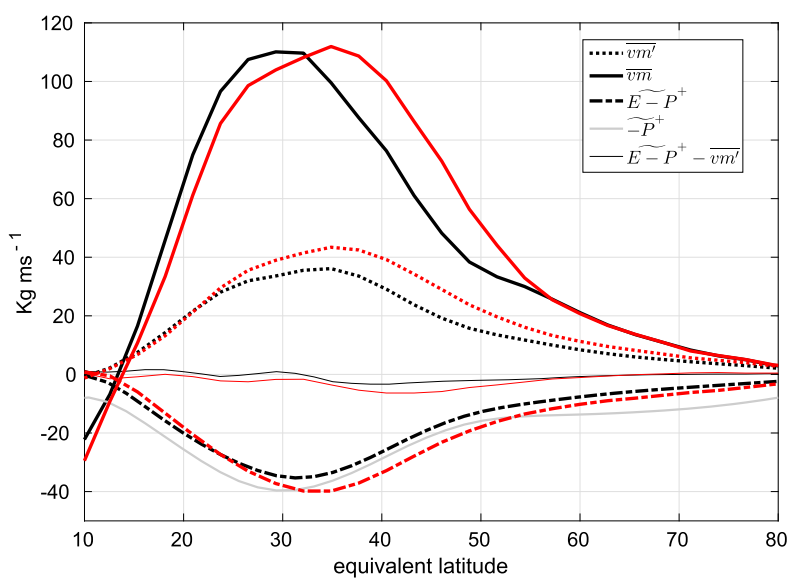

FIG. 10. The CWV wave activity budget for the moist area consisting of $\overline{v m}$ (black solid line), $\overline{v m^{\prime}}$ (black dotted line), and $\overline{(E-P)}^{+}$(black dashed-dotted line). See text for the detailed explanation of the terms. The corresponding terms from the 3-K warming simulation are shown with red lines. The gray line denotes $\overline{(-P)}^{+}$in the control simulation, showing the small contribution from $\widetilde{(E)}^{+}$through the comparison with $\overline{(E-P)}^{+}$. The source (sink) terms are expressed as positive (negative) values in the diagram.

moist region without participating the hydrological cycle. Thus, only a portion of $\overline{v m}^{+}$is involved in the hydrological cycle and converted into precipitation within the moist areas; as such, a ratio $\frac{v^{\prime}}{}+1 \overline{v m}^{+}$can be defined to represent precipitation efficiency provided that the contribution of evaporation is small and hence negligible (this turns out to be the case in the subtropics and midlatitudes):

$$
\frac{{\overline{v m^{\prime}}}^{+}}{\overline{v m}^{+}} \approx \frac{\overline{(P-E)}^{+}}{\overline{v m}^{+}} \text {. }
$$

The result of the budget based on the control MPAS simulation is reported in Fig. 10. It is clear that the $\overline{v m}^{+}$ term (or headwater influx; black solid line) dominates other terms in the budget. Further the direct calculation indeed verifies the balance between $\overline{(P-E)}^{+}$and ${\overline{v m^{\prime}}}^{+}$, with a small residual shown as the thin line in Fig. 10. Over a wide latitudinal range from the tropics to the midlatitude, the precipitation efficiency is no greater than $35 \%$, with a minimum of about $30 \%$ at around $25^{\circ} \mathrm{N}$, the peak latitude of $A^{+}$and where ARs are most prevalent (see Fig. 13c). As such, the moisture transport near this equivalent latitude indeed possesses some river-like characteristics: on the one hand, a large proportion $(\sim 70 \%)$ of the water vapor gets transported along the "river" downstream without being leaked out via precipitation, evincing the transport function of rivers; on the other hand, the contribution from evaporation to the budget is rather secondary in the tropical-to-midlatitude 


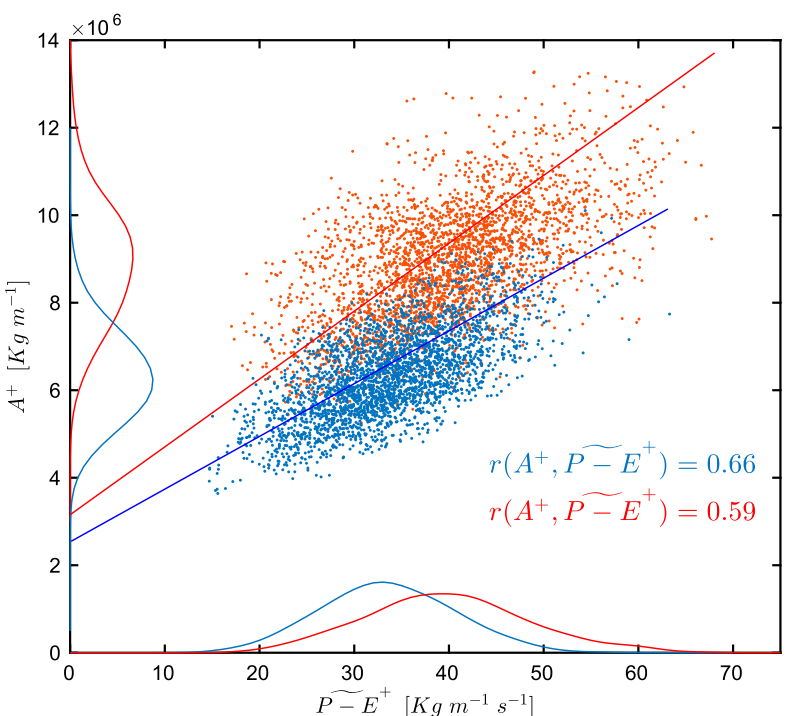

FIG. 11. The scatter of 6-hourly $A^{+}$against $\overline{(P-E)}^{+}$at $30^{\circ} \mathrm{N}$ and the corresponding normalized histograms (curves) from the control (blue) and 3-K (red) MPAS simulations. The intercept with the $y$ axis gives the value of $A_{c}^{+}$. The correlations between $A^{+}$and $\overline{(P-E)}^{+}$are also labeled.

regions [cf. $\overline{(P-E)}^{+}$and $\widetilde{(P)^{+}}$in Fig. 10] and thus the collection en route of water vapor via evaporation is small relative to other latitudes. Visual inspection of the time evolution of CWV and evaporation indicates that the features of ARs are often accompanied by minimum evaporation. This suggests that the lessened humidity difference between the lowest level of the atmosphere and the sea surface, owing to the warm, moist air plume blowing over the relatively cold sea surface, dominates over the effects of the large surface wind speed on surface evaporation underneath the atmospheric rivers. Some observational evidence from field campaigns also supports this notion (Bao et al. 2006).

Under $3-\mathrm{K}$ warming, the $\overline{v m}^{+}$term (an Eulerian quantity) is characterized by a poleward shift, reflecting the poleward shift of the Eulerian mean circulation and the zonal mean jet typical of the aquaplanet response to SST warming (e.g., Chen et al. 2013). This Eulerian shift is also manifested in the moist wave activity $A^{+}$and the associated sink $\overline{(P-E)}+$ and source ${\overline{v m^{\prime}}}^{+}$. However, in the latter two quantities the increase nearby and poleward of the mean jet exceeds markedly the decrease in the subtropics. Given the Lagrangian aspect of these terms (keep in mind that $m^{\prime}$ is the anomaly with respect to the Lagrangian $M$ ) and their closer association with the hydrological extremes, their response to SST warming unravels an intriguing difference in the response of the hydrological extremes compared to the
Eulerian mean hydrological cycle: the extremes are more characteristic of an intensification than a shift. This might underpin the large, often super-CC, increase of the precipitation extremes near and poleward of the storm track found in the future scenario simulations in CMIP5 (e.g., Toreti et al. 2013).

Similar to the $\overline{(P-E)} \sim A$ relationship, a significant linear relationship also exists between $\overline{(P-E)}$, and $A^{+}$ (see Fig. 11 for an example), providing a basis for scaling $(\overline{P-E})^{+}$with the information of wave activity. As now $A^{+}$is computed from the total CWV ( $m$ instead of $m^{\prime}$ ), an intercept results from linear regression between $A^{+}$ and $\overline{(P-E)^{+}}$and can be sensitive to which is treated as the independent variable. To avoid this ambiguity, a more objective empirical orthogonal function (EOF)based regression (e.g., York 1966) that minimizes the perpendicular distance from the regression line is utilized to estimate $\tau$ (slope) and $A_{c}^{+}$(intercept) in the relation below:

$$
\overline{(P-E)}^{+} \approx \frac{\left(A^{+}-A_{c}^{+}\right)}{\tau} .
$$

The meridional distributions of $A^{+}$and $A_{c}^{+}$are shown in Fig. 12a. Like the full wave activity $A, A^{+}$also peaks at approximately $25^{\circ}$ latitude, marking the locations of the activities associated with ARs. Note that $A_{c}^{+}$is smaller than $A^{+}$everywhere and can be thought of as the water vapor holding capacity of the atmosphere prior to saturation. In fact, (16) offers a new angle to interpret the hydrological cycle in the moist areas in quasiequilibrium: $P-E$ is operating in such a way so as to restore the CWV toward the capacity level $A_{c}^{+}$at time scale $\tau$, which itself can be quantified as the regression slope. The physical meaning of $A_{c}^{+}$can be further deciphered by scaling $A^{+}$as $-0.5 \eta^{+2} \partial M / \partial y$ and $A_{c}^{+}$as $-0.5 \eta_{c}^{+2} \partial M / \partial y$, respectively. As such, $\eta_{c}^{+}$assumes a meaning of "mean free path" of the air parcels within which the air parcels can travel freely poleward with the humidity content conserved, while $\eta^{+}$represents the actual distance traveled by the air parcels and the part beyond $\eta_{c}^{+}$(i.e., $\eta^{+}>\eta_{c}^{+}$) is deemed the distance over which condensation takes place. The meridional profiles of $\eta_{c}^{+}$and $\eta^{+}$are shown in Fig. 12b. The factors that determine the value of $\eta_{c}^{+}$and its variation with latitude can be complex, possibly involving the saturation deficit at origin, the magnitude and the slope of stirring, the cooling rate of the air parcels, and so on.

For a complete life cycle of an AR archetypical of the equilibrium picture, $A_{c}^{+}$can be interpreted as the part of moisture not actively participating in the hydrological cycle in the atmospheric river. Accordingly, the rate of conversion of $A^{+}-A_{c}^{+}$through $\left.\overline{(P-E)}\right)^{+}$ 

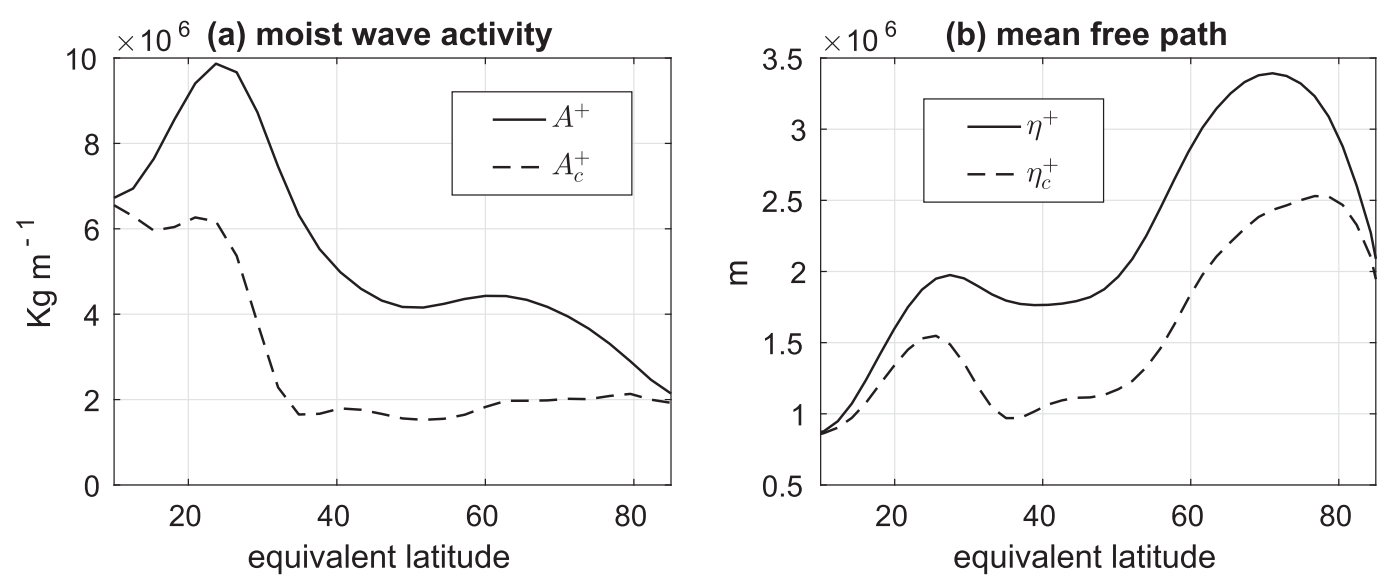

FIG. 12. The meridional distribution of (a) $A^{+}$and $A_{c}^{+}$and (b) the corresponding stirring scale $\eta^{+}$and mean free path $\eta_{c}^{+}$in the control MPAS simulation.

(i.e., $\tau^{-1}$ ) serves as a natural indicator for the rate of the hydrological cycle. The estimated time scale $\tau$ (Fig. 13a) displays a considerable variation with latitude, with a minimum of 1.5 days at about $25^{\circ}$ latitude where atmospheric rivers are most active. This minimum time scale and hence the highest hydrological cycling rate are the result of the combination of the minimum participation ratio of moisture $\left(A^{+}-A_{c}^{+}\right) / A^{+}$(Fig. 13b) and the relatively large $\overline{(P-E)^{+}}$there (see black dotteddashed line in Fig. 10). Together with the largest moist wave activity $A^{+}$and minimum precipitation efficiency, these characteristics define the uniqueness of the atmospheric rivers.

Following suit as for $\overline{(P-E)}$, we may construct a scaling for the fractional change of $(P-E)^{+}$forced by $3-\mathrm{K}$ warming based on (16):

$$
\frac{\delta \overline{(P-E)}}{\overline{(P-E)^{+}}} \approx \frac{\delta\left(A^{+}-A_{c}^{+}\right)}{\left(A^{+}-A_{c}^{+}\right)}+\frac{\delta \tau^{-1}}{\tau^{-1}} .
$$

The result of the scaling is summarized in Fig. 14. First, $\overline{(P-E)}^{+}$shows a super-CC rate of increase poleward of the mean jet, with a greater rate of increase than $\overline{(P-E)}$ (cf. Fig. 8a) at high latitudes. The fractional increase in $A^{+}-A_{c}^{+}$contributes positively almost everywhere to the increase of $\overline{(P-E)^{+}}$. Further inspection of moist wave activity $A^{+}$and water vapor holding capacity $A_{c}^{+}$ separately shows an increase in both; but the former increase is at a greater rate than the later, resulting in a net increase in $A^{+}-A_{c}^{+}$(orange line, Fig. 14). The larger rate of $\overline{(P-E)}+$ increase compared to $\overline{(P-E)}$ at high latitudes may be partially accounted for by the intensification of the hydrological cycling rate $\tau^{-1}$ there. The change of $\tau^{-1}$ features an overall dipolar structure, with a reduction equatorward and an intensification poleward of $47^{\circ}$ latitude. Compared to the weakening of the hydrological intensity for the total wave activity $A$ (Fig. 8e), the poleward intensification here implies a significant asymmetry in the hydrological cycle between the moist and dry areas.

\section{Summary and discussion}

The transient aspects of the atmospheric hydrological cycle and the related extremes pose challenges to the community in terms of understanding their fundamental structural constraints and their response to climate warming. Inspired by the recent development of the concept and budget of finite-amplitude wave activity in geophysical fluid dynamics, we construct a contourfollowing framework to analyze the hydrological budget following the CWV features by an area-coordinate transformation of the CWV equation. The resultant wave activity budget equation presents an areaintegrated moisture budget for areas districted by the isolines of the CWV or, effectively, for different climate regions stratified according to the CWV level. The immediate benefit of this approach is the simplicity in the formalism of the budget compared to its Eulerian equivalent (i.e., the CWV variance equation). The sensitivity of the budget to model resolution has also been examined by doubling the horizontal resolution twice (resulting in grid cells of nominal sizes of 120 and $60 \mathrm{~km}$, respectively), but no qualitative differences are found and hence not reported.

Notably, the resultant wave activity and its major sinks and sources become symmetrically or even normally distributed, and thus more tractable by linear analysis. A principal linear relationship emerges between the transformed $P-E$-or $\overline{(P-E)}$ measuring dry-versus-wet disparity-and the wave activity for each equivalent latitude, with their regression slope 
(a) $\tau$

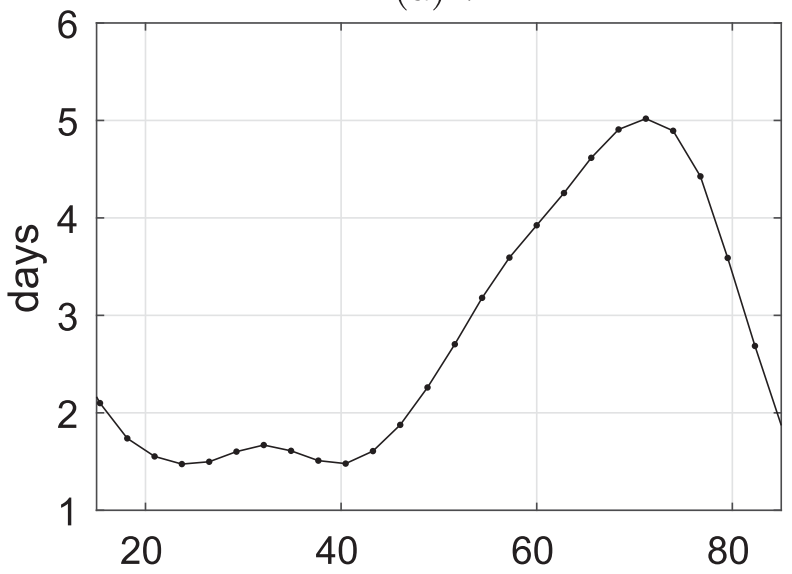

(b) $\left(A^{+}-A_{c}^{+}\right) / A^{+}$

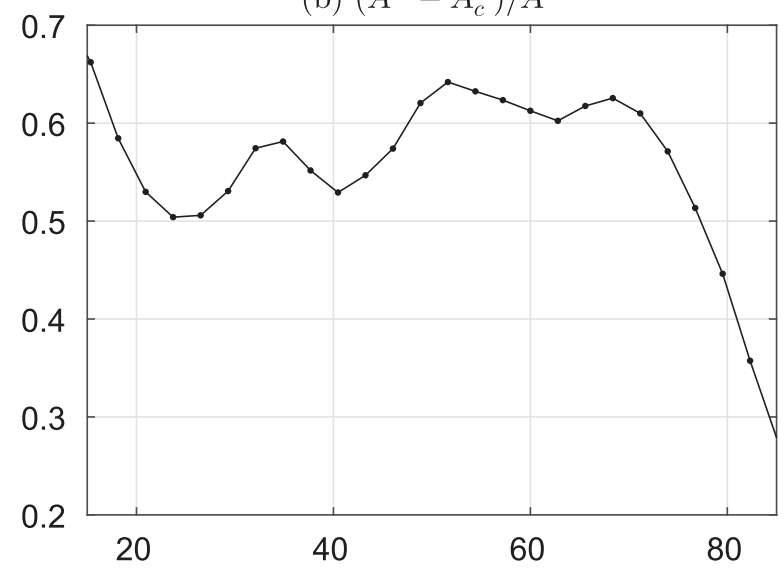

(c) $\overline{v m^{\prime}} / \overline{v m}$

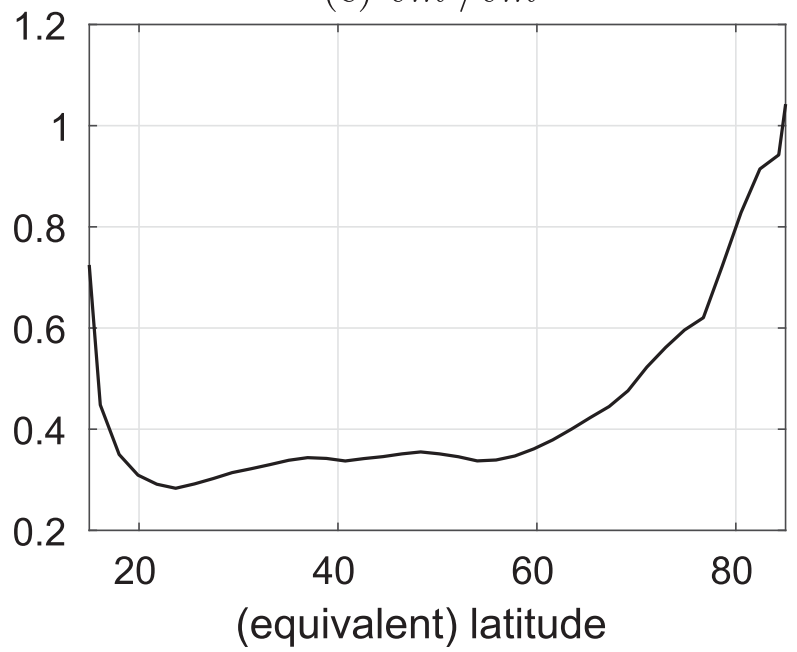

FIG. 13. (a) The regression slope of $A^{+}$against $\overline{(P-E)}^{+}$, of which the reciprocal $1 / \tau$ indicates the intensity of hydrological cycle. (b) CWV participation rate measured by $\left(A^{+}-A_{c}^{+}\right) / A^{+}$. (c) Precipitation efficiency ${\overline{v m^{\prime}}}^{+} / \overline{v m}^{+} \approx \overline{(P-E)}^{+} / \overline{v m}^{+}$. See text for detailed explanation of the terms. representing the rate of the hydrological cycle for the transient moisture waves. This relationship can be employed to form a scaling for the fractional change of $\overline{(P-E)}$ under SST warming. It is found that the meridional structure of $\delta \overline{(P-E)} / \overline{(P-E)}$ is largely dominated by the change of the stirring scale $\eta$, a dynamic factor reflecting the effect of the modulated Rossby wave breakings under SST warming. While the change of residence time $\tau$ also shows some meridional variations, largely due to the poleward shift of the mean profile, it is characteristic more of an overall increase in the extratropics, indicating an overall weakening of the rate of the eddy hydrological cycle-the most notable result of this study.

The wave activity analysis can be readily extended to address the budget only over the moist regions of the globe. In so doing, the resultant budget represents the moisture budget following the moist sectors of the weather systems. The maximum moist wave activity picks out naturally the equivalent latitude where atmospheric rivers are most prevalent (at $\sim 25^{\circ}$ latitude in the aquaplanet model examined). The budget analysis reveals some unique characteristics of the atmospheric rivers compared with other equivalent latitudes: (i) minimum participation ratio of moisture in the hydrological cycle, epitomizing the transport function of the atmospheric rivers; (ii) minimum precipitation efficiency; and (iii) maximum hydrological cycle rate. In response to a 3-K SST warming, $\overline{(P-E)}+$ increases at a super-CC rate in the extratropics, more characteristic of an intensification than poleward shift compared to the zonal mean $P-E$. In the meantime, the scaling for $\overline{(P-E)}^{+}$reveals a large reduction in the rate of hydrological cycle near the latitudes of ARs and an increase at mid-to-high latitudes, the latter being possibly the result of change of skewness in the vertical motion. As discussed in Pendergrass and Gerber (2016), the increase in the positive skewness in the vertical velocity might be the most important factor in modulating the probability distribution of precipitation toward more frequent precipitation extremes. In the framework of finite-amplitude wave activity, the skewness can be measured by the asymmetry between the moist and dry wave activities. Moreover, considerable asymmetry has been found between the changes in the vertical motion over the moist areas and dry areas under $3-\mathrm{K}$ warming perturbation (not shown). The cause for this asymmetry is likely fundamental to the moist dynamics of the midlatitude atmosphere, an important topic for future investigation.

Although we see a resemblance between the weakening of the transient hydrological cycling rate and that of the global mean hydrological cycle rate under global 


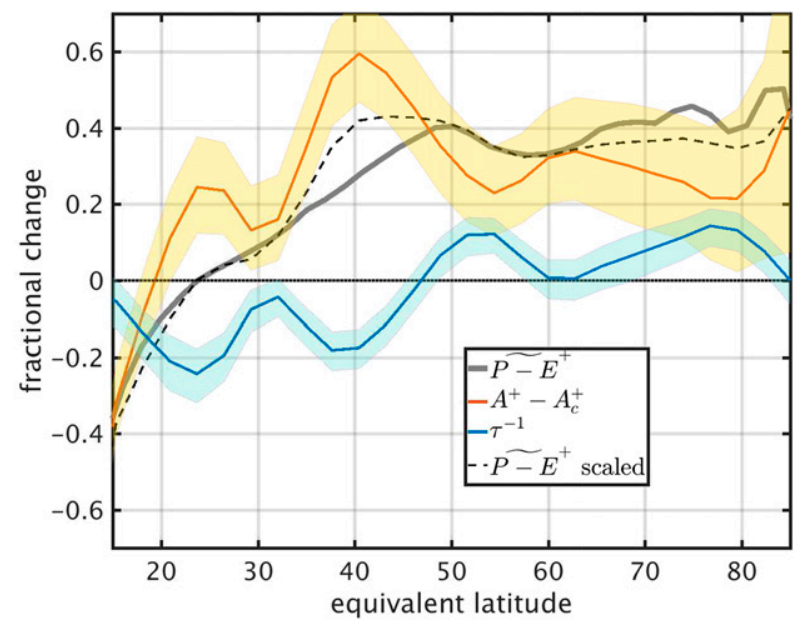

FIG. 14. Result of scaling using (17). The fractional change $\delta \overline{(P-E)}^{+} / \overline{(P-E)}^{+}$(gray line) can be scaled as the sum of (i) change of participation wave activity $\delta\left(A^{+}-A_{c}^{+}\right) /\left(A^{+}-A_{c}^{+}\right)$ (orange line) and (ii) change of hydrological cycle intensity $\delta \tau^{-1} / \tau^{-1}$ (blue line). The sum of (i) and (ii) is shown as the black dashed line. The color shading denotes the largest possible range of uncertainty arising from the regression uncertainties in $\tau$ and $A_{c}^{+}$(represented by their $95 \%$ confidence intervals) in the control and $3-\mathrm{K}$ simulations. In the estimate of the uncertainty range of $\delta\left(A^{+}-A_{c}^{+}\right) /\left(A^{+}-A_{c}^{+}\right)$, only the uncertainties in $A_{c}^{+}$are considered.

warming, the exact mechanism behind the former remains elusive and warrants further investigation. Guided by the working hypothesis of an energetic constraint (as for the global mean hydrological cycle), ongoing effort is concentrating on a wave activity budget for moist static energy to link the hydrological cycle to the energy cycle and the result will be reported elsewhere.

It should be stressed that the extratropical hydrological cycle as examined here is mostly about the modelresolved component and governed by the resolved atmospheric circulation, and thus should be less sensitive (compared to the tropical hydrological cycle) to the uncertainties in the convection parameterization of the model. Some confidence may be placed in the qualitative aspects of the many assertions reached above, whereas the quantitative aspects might vary with model resolution and configuration, a sensitivity in need of further investigation.

Despite the idealization in the current study, many broad features revealed herein can have important implications to what would happen in the real world under global warming. For instance, the muted rate of increase of the zonal mean $P-E$ compared to the increase in the wave $\overline{(P-E)}^{+}$is consistent with the finding from CMIP5 models that precipitation extremes increase at a greater rate than the mean precipitation under future warming scenarios (e.g., Pall et al. 2007; O'Gorman and Schneider 2009). In addition, the dipole in the change of the stirring scale under $3-\mathrm{K}$ warming echoes strongly the dipole in the circulation-induced changes in the frequency of atmospheric rivers found in CMIP5 simulations under the representative concentration pathway 8.5 emission scenario (e.g., Gao et al. 2016). A parallel effort is ongoing to bridge the gap between the aquaplanet world and the real world by extending the same analysis to the data output from CMIP5 climate model experiments.

Acknowledgments. We thank Takeshi Horinouchi and two anonymous reviewers for their perceptive comments on an earlier version of the manuscript. This study is supported by the U.S. Department of Energy Office of Science Biological and Environmental Research (BER) as part of the Regional and Global Climate Modeling program. PNNL is operated for DOE by Battelle Memorial Institute under Contract DE-AC05-76RL01830. GC is supported by the DOE Grant DE-SC0016117. ES is supported by the DOE Grant DE-SC0012599.

\section{APPENDIX}

\section{The Dominant Balance in Moist Areas}

For equilibrium, (14) becomes

$$
-\overline{v m}^{+}=\overline{(E-P)}^{+}-\frac{1}{2 \pi a \cos \phi_{e}} \int_{\tilde{L}}(\mathbf{v} m) \cdot \mathbf{n} d l,
$$

and it can be further written as

$$
\overline{(P-E)}^{+}-{\overline{v m^{\prime}}}^{+}=\overline{v M}^{+}-\frac{1}{2 \pi a \cos \phi_{e}} \int_{\tilde{L}}\left[\mathbf{v}\left(M+m^{\prime}\right)\right] \cdot \mathbf{n} d l,
$$

with $m$ being decomposed into $M$ and a deviation therefrom, $m^{\prime}$. Note that the time average has been applied to each term of the equations above, but is not shown for the compactness of mathematical expression. By noting the fact that $m=M$ and hence $m^{\prime}=0$ on $\tilde{L}$ and that $\tilde{L}+\bar{L}$ forms a close loop for line integral, we can express (A2) as

$$
\overline{(P-E)}^{+}-{\overline{v m^{\prime}}}^{+}=-\frac{M}{2 \pi a \cos \phi_{e}} \oint_{\tilde{L}+\bar{L}} \mathbf{v} \cdot \mathbf{n} d l .
$$

Using divergence theorem, the right-hand side of (A3) becomes 


$$
-\frac{M}{2 \pi a \cos \phi_{e}} \iint_{m>M, \phi>\phi_{e}}(\nabla \cdot \mathbf{v}) d S .
$$

If $m$ were a conservative tracer and $M$ an impermeable interface, the area integral above would be identically zero. Although neither condition is true for column integrated water vapor, the term on the right-hand side of (A3) is small compared to each of the terms on the lefthand side, to the extent that the water vapor weighted velocity $\mathbf{v}$ is dominated by the rotational component (i.e., $\left.\left|\mathbf{v}_{\psi}\right| \gg\left|\mathbf{v}_{\chi}\right|\right)$ in the extratropics. Indeed, computed as the residual of the two terms on the left-hand side of (A3), it is small (thin lines in Fig. 10). For the budget areas in subject, $\mathbf{v} M$ represents the part of the transport that passes through the areas without participating the hydrological cycle.

\section{REFERENCES}

Allen, M. R., and W. J. Ingram, 2002: Constraints on future changes in the hydrologic cycle. Nature, 419, 224-232, doi:10.1038/ nature 01092 .

Bao, J.-W., S. A. Michelson, P. J. Neiman, F. M. Ralph, and J. M. Wilczak, 2006: Interpretation of enhanced integrated water vapor bands associated with extratropical cyclones: Their formation and connection to tropical moisture. Mon. Wea. Rev., 134, 1063-1080, doi:10.1175/MWR3123.1.

Barnes, E. A., and D. L. Hartmann, 2012: Detection of Rossby wave breaking and its response to shifts of the midlatitude jet with climate change. J. Geophys. Res., 117, D09117, doi:10.1029/2012JD017469.

Chen, G., J. Lu, and L. Sun, 2013: Delineating the eddy-zonal flow interaction in the atmospheric response to climate forcing: Uniform SST warming. J. Atmos. Sci., 70, 22142233, doi:10.1175/JAS-D-12-0248.1.

Collins, M., and Coauthors, 2013: Long-term climate change: Projections, commitments and irreversibility. Climate Change 2013: The Physical Science Basis, T. F. Stocker et al., Eds., Cambridge University Press, 1029-1136, doi:10.1017/ CBO9781107415324.024.

Du, Q., V. Faber, and M. Gunzburger, 1999: Centroidal Voronoi tessellations: Application and algorithms. SIAM Rev., 41, 637676, doi:10.1137/S0036144599352836.

Emori, S., and S. Brown, 2005: Dynamic and thermodynamic changes in mean and extreme precipitation under changed climate. Geophys. Res. Lett., 32, L17706, doi:10.1029/ 2005 GL023272.

— - A. Hasegawa, T. Suzuki, and K. Dairaku, 2005: Validation, parameterization dependence, and future projection of daily precipitation simulated with a high-resolution atmospheric GCM. Geophys. Res. Lett., 32, L06708, doi:10.1029/ 2004GL022306.

Galewsky, J., A. Sobel, and I. Held, 2005: Diagnosis of subtropical humidity dynamics using tracers of last saturation. J. Atmos. Sci., 62, 3353-3367, doi:10.1175/JAS3533.1.

Gao, Y., L. R. Leung, J. Lu, and G. Masato, 2015a: Persistent cold air outbreaks over North America in a warming climate. Environ. Res. Lett., 10, 044001, doi:10.1088/1748-9326/10/4/ 044001.

— J. Lu, L. R. Leung, Q. Yang, S. Hagos, and Y. Qian, 2015b: Dynamical and thermodynamical modulations on future changes of landfalling atmospheric rivers over North America. Geophys. Res. Lett., 42, 7179-7186, doi:10.1002/ 2015 GL065435.

,$- \ldots$, and — 2016: Uncertainties in projecting future changes in atmospheric rivers and their impacts on heavy precipitation over Europe. J. Climate, 29, 6711-6726, doi:10.1175/JCLI-D-16-0088.1.

Haynes, P., 2010: Transport and mixing of atmospheric pollutants. Environmental Hazards: The Fluid Dynamics and Geophysics of Extreme Events, H. K. Moffatt and E. Shuckburgh, Eds., Lecture Note Series, Vol. 21, World Scientific, 155-192.

Held, I. M., and B. J. Soden, 2006: Robust response of the hydrological cycle to global warming. J. Climate, 19, 5686-5699, doi:10.1175/JCLI3990.1.

Hennessy, K., J. Gregory, and J. Mitchell, 1997: Changes in daily precipitation under enhanced greenhouse conditions. Climate Dyn., 13, 667-680, doi:10.1007/s003820050189.

Ingram, W., 2002: On the robustness of the water vapor feedback: GCM vertical resolution and formulation. J. Climate, 15, 917-921, doi:10.1175/1520-0442(2002)015<0917: OTROTW $>2.0 . \mathrm{CO} ; 2$.

Kharin, V. V., and F. W. Zwiers, 2000: Changes in the extremes in an ensemble of transient climate simulations with a coupled atmosphere-ocean GCM. J. Climate, 13, 3760-3788, doi:10.1175/ 1520-0442(2000)013<3760:CITEIA > 2.0.CO;2.

, _- X. Zhang, and M. Wehner, 2013: Changes in temperature and precipitation extremes in the CMIP5 ensemble. Climatic Change, 119, 345-357, doi:10.1007/s10584-013-0705-8.

Lau, W. K. M., and K.-M. Kim, 2015: Robust Hadley circulation changes and increasing global dryness due to $\mathrm{CO}_{2}$ warming from CMIP5 model projections. Proc. Natl. Acad. Sci. USA, 112, 3630-3635, doi:10.1073/pnas.1418682112.

Lenderink, G., and E. van Meijgaard, 2008: Increases in hourly precipitation extremes beyond expectations from temperature changes. Nat. Geosci., 1, 511-514, doi:10.1038/ ngeo262.

Leung, L. R., T. Ringler, W. D. Collins, M. Taylor, and M. Ashfaq, 2013: A hierarchical evaluation of regional climate simulations. Eos, Trans. Amer. Geophys. Union, 94, 297-298, doi:10.1002/2013EO340001.

Lu, J., R. L. Leung, Q. Yang, G. Chen, W. D. Collins, F. Li, Z. J. Hou, and X. Feng, 2014a: The robust dynamical contribution to precipitation extremes in idealized warming simulations across model resolutions. Geophys. Res. Lett., 41, 2971-2978, doi:10.1002/2014GL059532.

, L. Sun, Y. Wu, and G. Chen, 2014b: The role of subtropical irreversible PV mixing in the zonal mean circulation response to global warming-like thermal forcing. J. Climate, 27, 22972316, doi:10.1175/JCLI-D-13-00372.1.

- G. Ghen, L. R. Leung, D. A. Burrow, Q. Yang, K. Sakaguchi, and S. Hagos, 2015: Toward the dynamical convergence on the jet stream in aquaplanet AGCMs. J. Climate, 28, 6763-6782, doi:10.1175/JCLI-D-14-00761.1.

Mitchell, J., C. Wilson, and W. Cunnington, 1987: On $\mathrm{CO}_{2}$ climate sensitivity and model dependence of results. Quart. J. Roy. Meteor. Soc., 113, 293-322, doi:10.1002/qj.49711347517.

Nakamura, N., and A. Solomon, 2010: Finite-amplitude wave activity and mean flow adjustments in the atmospheric general circulation. Part I: Quasigeostrophic theory and analysis. J. Atmos. Sci., 67, 3967-3983, doi:10.1175/2010JAS3503.1.

, and D. Zhu, 2010: Finite-amplitude wave activity and diffusive flux of potential vorticity in eddy-mean flow interaction. J. Atmos. Sci., 67, 2701-2716, doi:10.1175/2010JAS3432.1. 
Neale, R. B., and B. J. Hoskins, 2000: A standard test for AGCMs including their physical parameterizations. I: The proposal. Atmos. Sci. Lett., 1, 101-107, doi:10.1006/asle.2000.0022.

— , and Coauthors, 2010: Description of the NCAR Community Atmosphere Model (CAM 4.0). NCAR Tech. Note NCAR/ TN-485+STR, 212 pp.

O'Brien, T. A., F. Li, W. D. Collins, S. A. Rauscher, T. D. Ringler, M. Taylor, S. M. Hagos, and L. R. Leung, 2013: Observed scaling in clouds and precipitation and scale incognizance in regional to global atmospheric models. J. Climate, 26, 93139333, doi:10.1175/JCLI-D-13-00005.1.

O'Gorman, P. A., and T. Schneider, 2006: Stochastic models for the kinematics of moisture transport and condensation in homogeneous turbulent flows. J. Atmos. Sci., 63, 2992-3005, doi:10.1175/JAS3794.1.

_, and _ 2009: The physical basis for increases in precipitation extremes in simulations of 21st-century climate change. Proc. Natl. Acad. Sci. USA, 106, 14773-14777, doi:10.1073/pnas.0907610106.

— , and C. J. Muller, 2010: How closely do changes in surface and column water vapor follow Clausius-Clapeyron scaling in climate-change simulations? Environ. Res. Lett., 5, 025207, doi:10.1088/1748-9326/5/2/025207.

Pall, P., M. R. Allen, and D. A. Stone, 2007: Testing the ClausiusClapeyron constraint on changes in extreme precipitation under $\mathrm{CO}_{2}$ warming. Climate Dyn., 28, 351-363, doi:10.1007/ s00382-006-0180-2.

Payne, A. E., and G. Magnusdottir, 2014: Dynamics of landfalling atmospheric rivers over the North Pacific in 30 years of MERRA reanalysis. J. Climate, 27, 7133-7150, doi:10.1175/ JCLI-D-14-00034.1.

Pendergrass, A., and E. Gerber, 2016: The rain is askew: Two idealized models relating vertical velocity and precipitation distributions in a warming world. J. Climate, 29, 6445-6462, doi:10.1175/JCLI-D-16-0097.1.

Pierrehumbert, R. T., and R. Roca, 1998: Evidence for control of Atlantic subtropical humidity by large scale advection. Geophys. Res. Lett., 25, 4537-4540, doi:10.1029/ 1998GL900203.

Rauscher, S. A., T. D. Ringler, W. C. Skamarock, and A. A. Mirin, 2013: Exploring a global multiresolution modeling approach using aquaplanet simulations. J. Climate, 26, 2432-2452, doi:10.1175/JCLI-D-12-00154.1.

Ringler, T., L. Ju, and M. Gunzburger, 2008: A multiresolution method for climate system modeling: Application of spherical centroidal Voronoi tessellations. Ocean Dyn., 58, 475-498, doi:10.1007/s10236-008-0157-2.
—, D. Jacobsen, M. Gunzburger, L. Ju, M. Duda, and W. C. Skamarock, 2011: Exploring a multiresolution modeling approach within the shallow-water equations. Mon. Wea. Rev., 139, 3348-3368, doi:10.1175/MWR-D-10-05049.1.

Rivière, G., 2011: A dynamical interpretation of the poleward shift of the jet streams in global warming scenarios. J. Atmos. Sci., 68, 1253-1272, doi:10.1175/2011JAS3641.1.

Royston, J. P., 1982: An extension of Shapiro and Wilk's W test for normality to large samples. J. Roy. Stat. Soc., 31C, 115-124.

Sakaguchi, K., and Coauthors, 2015: Exploring a multiresolution approach using AMIP simulations. J. Climate, 28, 5549-5574, doi:10.1175/JCLI-D-14-00729.1.

Schneider, T., T. Bischoff, and H. Płotka, 2015: Physics of changes in synoptic midlatitude temperature variability. J. Climate, $\mathbf{2 8}$, 2312-2331, doi:10.1175/JCLI-D-14-00632.1.

Semenov, V., and L. Bengtsson, 2002: Secular trends in daily precipitation characteristics: Greenhouse gas simulation with a coupled AOGCM. Climate Dyn., 19, 123-140, doi:10.1007/ s00382-001-0218-4.

Skamarock, W. C., and A. Gassmann, 2011: Conservative transport schemes for spherical geodesic grids: High-order flux operators for ODE-based time integration. Mon. Wea. Rev., 139, 2962-2975, doi:10.1175/MWR-D-10-05056.1.

Toreti, A., and Coauthors, 2013: Projections of global changes in precipitation extremes from Coupled Model Intercomparison Project phase 5 models. Geophys. Res. Lett., 40, 4887-4892, doi:10.1002/grl.50940.

Trenberth, K. E., and C. J. Guillemot, 1995: Evaluation of the global atmospheric moisture budget as seen from analyses. J. Climate, 8, 2255-2272, doi:10.1175/1520-0442(1995)008<2255: EOTGAM $>2.0 . \mathrm{CO} ; 2$.

—, A. Dai, R. M. Rasmussen, and D. B. Parsons, 2003: The changing character of precipitation. Bull. Amer. Meteor. Soc., 84, 1205-1217, doi:10.1175/BAMS-84-9-1205.

Wehner, M., 2004: Predicted twenty-first-century changes in seasonal extreme precipitation events in the Parallel Climate Model. J. Climate, 17, 4281-4290, doi:10.1175/JCLI3197.1.

Wright, J. S., A. Sobel, and J. Galewsky, 2010: Diagnosis of zonal mean relative humidity in a warmer climate. J. Climate, $\mathbf{2 3}$, 4556-4569, doi:10.1175/2010JCLI3488.1.

York, D., 1966: Least-squares fitting of a straight line. Can. J. Phys., 44, 1079-1086, doi:10.1139/p66-090.

Zhu, Y., and R. E. Newell, 1998: A proposed algorithm for moisture fluxes from atmospheric rivers. Mon. Wea. Rev., 126, 725-735, doi:10.1175/1520-0493(1998)126<0725:APAFMF>2.0.CO;2. 